\title{
Atmospheric sound propagation over large-scale irregular terrain
}

\author{
Martin Almquist*1, Ilkka Karasalo ${ }^{\dagger 2}$ and Ken Mattsson ${ }^{\ddagger 1}$ \\ ${ }^{1}$ Department of Information Technology, Uppsala University \\ ${ }^{2}$ Department of Hydroacoustics, Swedish Defence Research Agency, \\ SE 17290 Stockholm, Sweden
}

September 16, 2013

\begin{abstract}
A benchmark problem on atmospheric sound propagation over irregular terrain has been solved using a stable fourth-order accurate finite difference approximation of a high-fidelity acoustic model. A comparison with the parabolic equation method and ray tracing methods is made. The results show that ray tracing methods can potentially be unreliable in the presence of irregular terrain.
\end{abstract}

Key words: wave propagation; irregular terrain; high-order finite difference methods

\section{Introduction}

High-fidelity simulations of sound propagation require an accurate treatment of the medium of propagation. In the atmosphere the speed of sound typically varies in space, which causes refraction of sound rays. As sound waves hit the surface of the earth they are partly reflected; the degree and direction of reflection depend on the topography and the type of ground. Measurements have shown that in order to accurately predict sound pressure levels (SPL), it is important to take the properties of both the atmosphere and the underlying terrain into account (see for example [40, 24]). In realistic applications, the atmospheric variations can be complex and the terrain is often irregular, which means both that the topography is non-trivial and that the type of ground can vary.

\footnotetext{
*martin.almquist@it.uu.se; Corresponding author

$\dagger^{\dagger}$ ilkka.karasalo@foi.se

${ }^{\ddagger}$ ken. mattsson@it.uu. se
} 
One high-fidelity model for sound propagation is based on the linearized Euler equations [41], which is most often solved with the staggered grid finite difference time domain method originally presented in [48]. In [17] a pseudo-spectral time domain method was used to solve the linearized Euler equations in urban courtyards. This is an efficient approach when the underlying geometry fits into a block-Cartesian geometry.

Another high-fidelity model is the time-dependent acoustic wave equation. However, the wave equation has been considered too computationally demanding in realistic large-scale $3-\mathrm{D}$ settings. One way to reduce the computational cost when the sound source consists of a single frequency is to Fourier transform the wave equation, which yields the Helmholtz equation. From the Helmholtz equation, it is possible to further reduce the computational cost via an assumption that reflections at boundaries are negligible. The resulting model is called the parabolic equation (PE) model [25], often used in ocean acoustics. Because of the assumption about reflections, the PE method is only valid for very moderate topographies. A significant drawback with the Helmholtz equation (and the PE method) is that timedependent phenomena, such as a varying atmosphere or sources (for instance wind turbines) can not easily be approximated.

However, despite the possible simplifications, the above models are all generally regarded as too computationally expensive in realistic 3-D applications. Hence, most numerical methods for sound propagation are based on much simpler models. A commonly used sound propagation model is NORD2000 [1], based on ray tracing methods, which combine geometrical ray theory with the theory of diffraction (for examples of applications see $[46,47,23,8])$. Ray tracing methods are computationally cheap, but they incorporate neither a complex atmosphere nor irregular terrain properly.

Unfortunately, due to a shortage of reliable measurements over largescale domains, it has been difficult to validate the sound propagation models currently used in practical applications. An alternative to measurements is validation through manufactured solutions (where one compares the numerical results with analytical solutions), but this technique has not yet been widely accepted outside the field of applied mathematics.

In this paper we shall solve a realistic sound propagation problem with the time-dependent acoustic wave equation as the underlying model. As mentioned above, this model is desirable from an accuracy perspective, but it is computationally costly. It is therefore imperative to use an efficient numerical method, to minimize the number of unknowns. It is well known that high-order (higher than second order) spatially accurate finite difference schemes combined with high-order accurate time marching schemes are very well suited for wave propagation problems on large domains (see the pioneering paper by Kreiss and Oliger [22]). To guarantee an accurate approximation, it is necessary that the underlying numerical scheme can be proven stable, which is a non-trivial task using high-order finite difference 
methods.

A robust and well-proven high-order finite difference methodology that ensures stability of time-dependent partial differential equations (PDEs) is the summation-by-parts-simultaneous approximation term (SBP-SAT) method. The SBP-SAT method combines semi-discrete operators that satisfy a summation-by-parts (SBP) formula [21], with physical boundary conditions implemented using the simultaneous approximation term (SAT) method [4]. Examples of the SBP-SAT approach can be found in[36, 37, 38, 32, 34, $35,39,30,44,26,7,33,14,13,16]$.

An added benefit of the SBP-SAT method is that it naturally extends to multi-block geometries while retaining the essential single-block properties: stability, accuracy, and conservation [5]. Thus, problems involving complex domains or non-smooth geometries are easily amenable to the approach. References [30, 34, 15, 29] report applications of the SBP-SAT method to problems involving nontrivial geometries. Most of the published results for the SBP-SAT method are for first order hyperbolic systems (and the NavierStokes equations). The extension of the SBP-SAT technique to the second order wave equation is found in $[30,31]$. However, those studies were restricted to 1-D concerning high-order finite difference methods (3-D results for the finite volume technique were presented). The present study is a direct extension of the SBP-SAT method to the second order wave equation on multidimensional curvilinear domains, including non-trivial boundary conditions.

In the first part of this paper, the SBP-SAT method is applied to the benchmark problem introduced in [40]. The SBP-SAT method is here extended to the second order wave equation on a curvilinear 2-D domain with non-trivial boundary conditions. A fourth-order accurate SBP-SAT approximation is implemented and verified by a grid-convergence study against manufactured solutions. In the second part of this paper, the benchmark problem in [40] is used to compare the PE and ray tracing methods with the newly implemented SBP-SAT method. Conclusions as to the validity of the $\mathrm{PE}$ and ray tracing methods in the presence of a complex atmosphere and irregular terrain are drawn.

The rest of the paper is organized as follows: In Section 2 we introduce some definitions and illustrate the SBP-SAT method by applying it to a 1-D problem. In Section 3 we analyze a model problem in 2-D. We then introduce the benchmark problem in Section 4, and describe how the SBPSAT technique has been adapted to this problem. The PE method and the ray interpolation methods are introduced in Sections 5 and 6, respectively. The implementation of the fourth-order accurate SBP-SAT method is verified in a series of convergence studies in Section 7. In Section 8, we present the results from the benchmark problem, comparing the different numerical methods. Conclusions are presented in Section 9. The finite difference (SBP) operators are listed in Appendix. 


\section{The 1-D problem}

In this section we define the SBP-SAT method. To illustrate the power and simplicity of the method we shall consider the following second-order hyperbolic problem:

$$
\begin{array}{lll}
a u_{t t}=\left(b u_{x}\right)_{x}, & 0 \leq x \leq 1, & t \geq 0, \\
L_{0} u=g_{0}(t), & x=0, & t \geq 0, \\
L_{1} u=g_{1}(t), & x=1, & t \geq 0, \\
u=f_{1}, \quad u_{t}=f_{2}, & 0 \leq x \leq 1, & t=0,
\end{array}
$$

where $a(x)>0$ and $b(x)>0$, and the boundary conditions are given by

$$
\begin{array}{ll}
L_{0} u=\alpha_{0} u_{t t}+\alpha_{1} u_{t}+\alpha_{2} u+\alpha_{3} u_{x}=g_{0}(t), & x=0 \\
L_{1} u=\beta_{0} u_{t t}+\beta_{1} u_{t}+\beta_{2} u+\beta_{3} u_{x}=g_{1}(t), & x=1 .
\end{array}
$$

In the present study we restrict ourselves to the case $\alpha_{3} \neq 0, \beta_{3} \neq 0$, referred to as mixed boundary conditions. For the treament of Dirichlet conditions we refer to [31].

Before we start employing the SBP-SAT method, some definitions are needed. Let the inner product for real-valued functions $u, v \in L^{2}[0,1]$ be defined by $(u, v)=\int_{0}^{1} u v c(x) d x, c(x)>0$, and let the corresponding norm be $\|u\|_{c}^{2}=(u, u)$. The domain $(0 \leq x \leq 1)$ is discretized using the following $N+1$ equidistant grid points:

$$
x_{i}=i h, \quad i=0,1, \ldots, N, \quad h=\frac{1}{N} .
$$

The approximate solution at grid point $x_{i}$ is denoted $v_{i}$, and the discrete solution vector is $v=\left[v_{0}, v_{1}, \ldots, v_{N}\right]^{T}$. Similar to the continuous inner product, we define an inner product for discrete real-valued vector functions $u, v \in \mathbf{R}^{\mathbf{N}+\mathbf{1}}$ by $(u, v)_{H_{c}}=u^{T} H C v$, where $H$ is diagonal and positive definite and $C$ is the projection of $c(x)$ onto the diagonal. The corresponding norm is $\|v\|_{H_{c}}^{2}=v^{T} H C v$.

Remark The matrix product $H C$ defines a norm if and only if $H C$ is symmetric and positive definite. This can only be guaranteed if $H$ is a diagonal matrix (see [43] for a detailed study on this).

The following vectors will be frequently used:

$$
e_{0}=[1,0, \ldots, 0]^{T}, \quad e_{N}=[0, \ldots, 0,1]^{T} .
$$

\subsection{The SBP-SAT method}

SBP operators are essentially central finite difference stencils, closed at the boundaries with carefully chosen one-sided difference stencils which mimic the underlying integration-by-parts formula in a discrete norm. In the present 
paper we address the SBP operators by the accuracy of the central scheme and the type of norm which they are based on. A $2 p$ th order diagonal norm SBP operator is closed with $p$ th order accurate one sided stencils (see [32]). For first order hyperbolic problems, this implies that the convergence rate (i.e., global convergence) drops to $(p+1)$ th order when using a $2 p$ th order diagonal norm SBP operator. For strongly parabolic problems and second order hyperbolic problems the convergence rate instead drops to $(p+2)$ th order (see $[10,45]$ for more information on the accuracy of finite difference approximations).

To define the SBP-SAT method, we present definitions 2.1-2.3 (first stated in [35] and [30]). We here say that a scheme is explicit if no linear system of equations needs to be solved to compute the difference approximation.

Definition 2.1 An explicit 2pth-order accurate finite difference stencil with minimal stencil width of a Cauchy problem is called a 2 pth-order accurate narrow-stencil.

Definition 2.2A difference operator $D_{1}=H^{-1} Q=H^{-1}\left(\bar{Q}-\frac{1}{2} e_{0} e_{0}^{T}+\right.$ $\left.\frac{1}{2} e_{N} e_{N}^{T}\right)$ approximating $\partial / \partial x$, using a 2 pth-order accurate narrow-stencil, is said to be a $2 p$ th-order accurate narrow-diagonal first-derivative SBP operator if $H$ is diagonal and positive definite and $\bar{Q}+\bar{Q}^{T}=0$.

Definition 2.3 Let $D_{2}^{(b)}=H^{-1}\left(-M^{(b)}-e_{0} b_{0} S_{0}+e_{N} b_{N} S_{N}\right)$ approximate $\partial / \partial x(b \partial / \partial x)$, where $b(x)>0$, using a 2 pth-order accurate narrow-stencil. $D_{2}^{(b)}$ is said to be a $2 p$ th-order accurate narrow-diagonal second-derivative SBP operator, if $H$ is diagonal and positive definite, $M^{(b)}$ is symmetric and positive semi-definite and $S_{0}$ and $S_{N}$ approximate the first-derivative operator at the boundaries.

The superscript $(b)$ emphasizes that $M^{(b)}$ and $D_{2}^{(b)}$ depend on $b(x)$. The explicit dependence can be found in [28]. For completeness we have included the fourth order SBP operators (used in the present study) in Appendix.

The following definition introduced in [28] is also central in this paper:

Definition 2.4 Let $D_{1}$ and $D_{2}^{(b)}$ be 2pth-order accurate narrow-diagonal first- and second-derivative SBP operators. If $M^{(b)}=D_{1}^{T} H B D_{1}+R_{(b)}$, and the remainder $R_{(b)}$ is positive semi-definite, $D_{1}$ and $D_{2}^{(b)}$ are called compatible.

\subsubsection{Continuous analysis}

Multiplying the first equation in (1) by $u_{t}$ and integrating by parts (referred to as "the energy method") leads to

$$
\frac{d}{d t}\left(\left\|u_{t}\right\|_{a}^{2}+\left\|u_{x}\right\|_{b}^{2}\right)=2\left(b u_{x} u_{t}\right)_{x=1}-2\left(b u_{x} u_{t}\right)_{x=0},
$$


where $\left(b u_{x} u_{t}\right)_{x=1}$ means $\left(b u_{x} u_{t}\right)$ evaluated at $x=1$. We also identify

$$
E=\left\|u_{t}\right\|_{a}^{2}+\left\|u_{x}\right\|_{b}^{2},
$$

as the total energy (kinetic and potential).

Multiplying the first equation in (1) by $u_{t}$, integrating by parts and imposing the boundary conditions (2) leads to

$$
\frac{d}{d t} \bar{E}=B T_{x=0}+B T_{x=1},
$$

where

$$
\bar{E}=\left\|u_{t}\right\|_{a}^{2}+\left\|u_{x}\right\|_{b}^{2}+\frac{b(0)}{\alpha_{3}}\left(\alpha_{0} u_{t}^{2}+\alpha_{2} u^{2}\right)_{x=0}-\frac{b(1)}{\beta_{3}}\left(\beta_{0} u_{t}^{2}+\beta_{2} u^{2}\right)_{x=0},
$$

and

$$
\begin{aligned}
& B T_{x=0}=+\frac{2 b(0) \alpha_{1}}{\alpha_{3}}\left(u_{t}-\frac{g_{0}}{2 \alpha_{1}}\right)_{x=0}^{2}-\frac{g_{0}^{2} b(0)}{2 \alpha_{1} \alpha_{3}} \\
& B T_{x=1}=-\frac{2 b(1) \beta_{1}}{\beta_{3}}\left(u_{t}-\frac{g_{1}}{2 \beta_{1}}\right)_{x=1}^{2}+\frac{g_{1}^{2} b(1)}{2 \beta_{1} \beta_{3}} .
\end{aligned}
$$

Here we assume that $\alpha_{3} \neq 0$ and $\beta_{3} \neq 0$. The following Lemma is central in the present study,

Lemma 2.5 Eq. (1) with boundary conditions (2) has a bounded energy in terms of initial and boundary data if $\alpha_{3} \neq 0, \beta_{3} \neq 0, \alpha_{0} \alpha_{3} \geq 0, \alpha_{2} \alpha_{3} \geq 0$, $\beta_{0} \beta_{3} \leq 0, \beta_{2} \beta_{3} \leq 0, \alpha_{1} \alpha_{3}<0$ and $\beta_{1} \beta_{3}>0$ hold.

Proof $\bar{E}$ is non-negative and well defined if $\alpha_{3} \neq 0, \beta_{3} \neq 0, \alpha_{0} \alpha_{3} \geq 0$, $\alpha_{2} \alpha_{3} \geq 0, \beta_{0} \beta_{3} \leq 0, \beta_{2} \beta_{3} \leq 0$ hold. By integrating (6) in time, we obtain

$$
\begin{gathered}
\bar{E}(t)+\int_{0}^{t}\left(\frac{2 b(1) \beta_{1}}{\beta_{3}}\left(u_{t}-\frac{g_{1}(\tau)}{2 \beta_{1}}\right)_{x=1}^{2}-\frac{2 b(0) \alpha_{1}}{\alpha_{3}}\left(u_{t}-\frac{g_{0}(\tau)}{2 \alpha_{1}}\right)_{x=0}^{2}\right) d \tau= \\
\bar{E}(0)+\int_{0}^{t}\left(\frac{g_{1}^{2}(\tau) b(1)}{2 \beta_{1} \beta_{3}}-\frac{g_{0}^{2}(\tau) b(0)}{2 \alpha_{1} \alpha_{3}}\right) d \tau .
\end{gathered}
$$

If $\alpha_{1} \alpha_{3}<0$ and $\beta_{1} \beta_{3}>0$ hold we have a strong estimate of $\bar{E}(t)$ in terms of initial and boundary data.

Remark The implication of Lemma 2.5 is that we can have at most linear time-growth of $\|u\|$. Linear growth (in $t$ ) does not violate well-posedness (see [11]). However, linear time-growth of $\|u\|$ can only occur if we have $f_{2} \neq 0$ combined with pure Neumann boundary conditions, i.e, $\alpha_{0}=\alpha_{1}=\beta_{0}=$ $\beta_{1}=0$, so that we have a zero eigenvalue in the spectrum [27]. 


\subsubsection{Semi-discrete analysis}

The discrete approximation of (1) using the SBP-SAT method is

$$
A v_{t t}=D_{2}^{(b)} v+\tau_{0} H^{-1} e_{0}\left(L_{0} v-g_{0}\right)+\tau_{1} H^{-1} e_{N}\left(L_{1} v-g_{1}\right),
$$

where $e_{0}$ and $e_{N}$ are defined in (3). (We assume the same initial conditions $v=f_{1}, \quad v_{t}=f_{2}$ as in the continuous case). The matrix $A$ has the values of $a(x)$ injected on the diagonal. The semi-discrete boundary operators that mimic (2) are given by

$$
\begin{aligned}
& L_{0} v=\alpha_{0}\left(v_{t t}\right)_{0}+\alpha_{1}\left(v_{t}\right)_{0}+\alpha_{2} v_{0}+\alpha_{3} S_{0} v \\
& L_{1} v=\beta_{0}\left(v_{t t}\right)_{N}+\beta_{1}\left(v_{t}\right)_{N}+\beta_{2} v_{N}+\beta_{3} S_{N} v .
\end{aligned}
$$

Applying the energy method by multiplying (9) by $v_{t}^{T} H$ and adding the transpose leads to

$$
\begin{aligned}
\frac{d}{d t} E_{H}= & -2 b_{0}\left(v_{t}\right)_{0} S_{0} v+2 b_{N}\left(v_{t}\right)_{N} S_{N} v \\
& +2 \tau_{0}\left(\alpha_{0}\left(v_{t}\right)_{0}\left(v_{t t}\right)_{0}+\alpha_{1}\left(v_{t}^{2}\right)_{0}\right) \\
& +2 \tau_{0}\left(\alpha_{2}\left(v_{t}\right)_{0} v_{0}+\alpha_{3}\left(v_{t}\right)_{0} S_{0} v-\left(v_{t}\right)_{0} g_{0}\right) \\
& +2 \tau_{1}\left(\beta_{0}\left(v_{t}\right)_{N}\left(v_{t t}\right)_{N}+\beta_{1}\left(v_{t}^{2}\right)_{N}\right) \\
& +2 \tau_{1}\left(\beta_{2}\left(v_{t}\right)_{N} v_{N}+\beta_{3}\left(v_{t}\right)_{N} S_{N} v-\left(v_{t}\right)_{N} g_{1}\right),
\end{aligned}
$$

where

$$
E_{H}=\left\|v_{t}\right\|_{H_{a}}^{2}+v^{T} M^{(b)} v .
$$

Lemma 2.6 Eq. (9) with boundary operators (10) exactly mimics the continuous energy estimate (6) if $\tau_{0}=\frac{b_{0}}{\alpha_{3}}, \tau_{1}=-\frac{b_{N}}{\beta_{3}}$, and is thus stable if the conditions in Lemma 2.5 hold.

Proof insert $\tau_{0}=\frac{b_{0}}{\alpha_{3}}, \tau_{1}=-\frac{b_{N}}{\beta_{3}}$ in (11) to obtain

$$
\frac{d}{d t} \bar{E}_{H}=B T_{0}+B T_{N}
$$

where

$\bar{E}_{H}=\left\|v_{t}\right\|_{H_{a}}^{2}+v^{T} M^{(b)} v+\frac{b_{0}}{\alpha_{3}}\left(\alpha_{0}\left(v_{t}^{2}\right)_{0}+\alpha_{2}\left(v^{2}\right)_{0}\right)-\frac{b_{N}}{\beta_{3}}\left(\beta_{0}\left(v_{t}^{2}\right)_{N}+\beta_{2}\left(v^{2}\right)_{N}\right)$,

and

$$
\begin{aligned}
& B T_{0}=+\frac{2 b_{0} \alpha_{1}}{\alpha_{3}}\left(\left(v_{t}\right)_{0}-\frac{g_{0}}{2 \alpha_{1}}\right)^{2}-\frac{g_{0}^{2} b_{0}}{2 \alpha_{1} \alpha_{3}} \\
& B T_{N}=-\frac{2 b_{N} \beta_{1}}{\beta_{3}}\left(\left(v_{t}\right)_{N}-\frac{g_{1}}{2 \beta_{1}}\right)^{2}+\frac{g_{1}^{2} b_{N}}{2 \beta_{1} \beta_{3}} .
\end{aligned}
$$

Equation (12) is a semi-discrete analogue to (6), and stability follows if the conditions in Lemma 2.5 hold. 


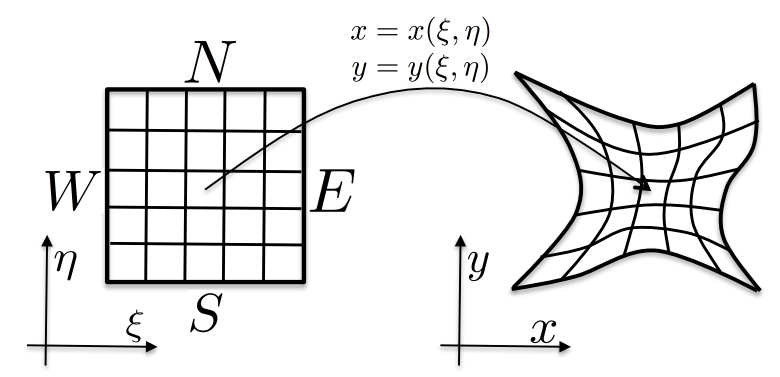

Figure 1: The mapping between cartesian (left) and curvilinear (right) coordinates

\section{Analysis in 2D}

In this section we analyze the scalar 2-D wave equation with mixed boundary conditions. To allow for complex domains, we transform the equation given on a curvilinear domain to an equation on a rectangular domain. We then derive an energy estimate for the continuous case. After discretizing the model in space with the SBP-SAT method, we prove stability by exactly mimicking the continuous energy estimate.

\subsection{Definitions}

To make the notation more compact we introduce the Kronecker product:

$$
C \otimes D=\left[\begin{array}{ccc}
c_{0,0} D & \cdots & c_{0, q-1} D \\
\vdots & & \vdots \\
c_{p-1,0} D & \cdots & c_{p-1, q-1} D
\end{array}\right],
$$

where $C$ is a $p \times q$ matrix and $D$ is an $m \times n$ matrix. We also let $I_{N}$ be the $(N+1) \times(N+1)$ identity matrix.

If the problem is given on a curvilinear domain $\Omega$ we transform it to the unit square, $\Omega^{\prime}$. We will refer to $\Omega$ as the physical domain and $\Omega^{\prime}$ as the rectangular domain. The rectangular domain is discretized using the $\left(N_{\xi}+1\right)\left(N_{\eta}+1\right)$ grid points:

$$
\left(\xi_{i}, \eta_{j}\right)=\left(\frac{i}{N_{\xi}}, \frac{j}{N_{\eta}}\right), \quad i=0,1, \ldots, N_{\xi}, \quad j=0,1, \ldots, N_{\eta} .
$$

The boundaries of $\Omega^{\prime}$ are denoted by $W$ (west), $N$ (north), $E$ (east) and $S$ (south), respectively, as shown in Figure 1. The approximate solution at a grid point $\left(\xi_{i}, \eta_{j}\right)$ is denoted by $v_{i j}$, and the discrete solution vector is $v=\left[v_{00}, \ldots, v_{0 N_{\eta}}, v_{10}, \ldots, v_{N_{\xi} N_{\eta}}\right]^{T}$. The matrix $R_{W}$ is defined so that $R_{W} v$ is a vector with the same length as $v$ and the same elements on the positions corresponding to the west boundary, but zeros everywhere else. The 
matrices $R_{N}, R_{E}$ and $R_{S}$ are defined similarly for the north, east and south boundaries, respectively.

By $D_{1 \xi}$ we denote the 2-D version of the narrow-stencil first-derivative SBP operator $D_{1}$, approximating $\frac{\partial}{\partial \xi}$. Similarly, $D_{2 \xi}^{(b)}$ approximates $\frac{\partial}{\partial \xi}\left(b \frac{\partial}{\partial \xi}\right)$. In the same manner, we let $H_{\xi}$ denote the 2-D version of the diagonal matrix $H$, applied in the $\xi$-direction. $D_{1 \eta}, D_{2 \eta}^{(b)}$ and $H_{\eta}$ are defined similarly for the $\eta$-direction.

To simplify the notation (without any restriction) we here assume $N_{\xi}=$ $N_{\eta}=N$. The 2-D operators can be neatly expressed in terms of the 1-D operators using the Kronecker product:

$$
\begin{array}{ll}
D_{1 \xi}=D_{1} \otimes I_{N}, & D_{1 \eta}=I_{N} \otimes D_{1} \\
H_{\xi}=H \otimes I_{N}, & H_{\eta}=I_{N} \otimes H \\
E_{W}=e_{0} \otimes I_{N}, & E_{S}=I_{N} \otimes e_{0} \\
R_{W}=E_{W} E_{W}^{T}, & R_{S}=E_{S} E_{S}^{T} \\
E_{E}=e_{N} \otimes I_{N}, & E_{N}=I_{N} \otimes e_{N}, \\
R_{E}=E_{E} E_{E}^{T}, & R_{N}=E_{N} E_{N}^{T},
\end{array}
$$

where the vectors $e_{0}$ and $e_{N}$ are defined in (3). Assuming that the coefficient $b$ is constant, we can also write

$$
D_{2 \xi}^{(b)}=D_{2}^{(b)} \otimes I_{N}, \quad D_{2 \eta}^{(b)}=I_{N} \otimes D_{2}^{(b)} .
$$

In the case of a variable coefficient $b$, however, (15) does not hold. To cover also that case, we introduce the notation

$$
b_{i}^{(\xi)}(\eta)=b\left(\xi_{i}, \eta\right), \quad b_{i}^{(\eta)}(\xi)=b\left(\xi, \eta_{i}\right), \quad i=0,1, \ldots, N .
$$

We also define

$$
d_{i j}^{(b)}=\left[\begin{array}{llll}
\left(D_{2}^{\left(b_{0}^{(\eta)}\right)}\right)_{i, j} & & & \\
& \left(D_{2}^{\left(b_{1}^{(\eta)}\right)}\right)_{i, j} & & \\
& & \ddots & \\
& & & \left(D_{2}^{\left(b_{N}^{(\eta)}\right)}\right)_{i, j}
\end{array}\right] .
$$

We then have

$$
D_{2 \xi}^{(b)}=\left[\begin{array}{cccc}
d_{11}^{(b)} & d_{12}^{(b)} & \cdots & d_{1(N+1)}^{(b)} \\
d_{21}^{(b)} & d_{22}^{(b)} & \cdots & d_{2(N+1)}^{(b)} \\
\vdots & \vdots & \ddots & \vdots \\
d_{(N+1) 1}^{(b)} & d_{(N+1) 2}^{(b)} & \cdots & d_{(N+1)(N+1)}^{(b)}
\end{array}\right]
$$


and

$$
D_{2 \eta}^{(b)}=\left[\begin{array}{cccc}
D_{2}^{\left(b_{0}^{(\xi)}\right)} & & & \\
& D_{2}^{\left(b_{1}^{(\xi)}\right)} & & \\
& & \ddots & \\
& & & D_{2}^{\left(b_{N}^{(\xi)}\right)}
\end{array}\right]
$$

\subsection{The continuous problem}

We consider the following problem:

$$
\begin{array}{lll}
u_{t t}=b \Delta u & (x, y) \in \Omega, & t \geq 0 \\
\gamma_{1} u+\gamma_{2} \nabla u \cdot \mathbf{n}+\gamma_{3} u_{t}=0 & (x, y) \in \partial \Omega, & t \geq 0 \\
u=f_{1}, \quad u_{t}=f_{2}, & (x, y) \in \Omega, & t=0,
\end{array}
$$

where $b(x, y)>0$. We have chosen homogeneous boundary conditions to avoid unnecessary notation in the analysis. Similarly to the 1-D analysis in Section 2.1, the analysis holds for inhomogeneous conditions as well. We also limit our present study to the case $\gamma_{2} \neq 0$, which includes the important case of Neumann conditions $\left(\gamma_{1}=0, \gamma_{2}=1, \gamma_{3}=0\right)$.

We can add dissipation to (20) by adding a term $b \nabla \cdot\left(\sigma \nabla u_{t}\right), \sigma(x, y) \geq 0$ to the right hand side of the PDE. The added dissipation term will be used to create absorbing layers at artificial boundaries in Section 4.1. Including the dissipation term, the problem reads

$$
\begin{array}{lll}
u_{t t}=b \Delta u+b \nabla \cdot\left(\sigma \nabla u_{t}\right) & (x, y) \in \Omega, & t \geq 0 \\
\gamma_{1} u+\gamma_{2} \nabla u \cdot \mathbf{n}+\gamma_{3} u_{t}=0 & (x, y) \in \partial \Omega, & t \geq 0 \\
u=f_{1}, \quad u_{t}=f_{2}, & (x, y) \in \Omega, & t=0 .
\end{array}
$$

We now transform the problem to a rectangular domain. Assume that there is a smooth one-to-one mapping

$$
\left\{\begin{array}{l}
x=x(\xi, \eta) \\
y=y(\xi, \eta)
\end{array}\right.
$$

from $\Omega^{\prime}$ to $\Omega$. The Jacobian $J$ of the transformation is

$$
J=x_{\xi} y_{\eta}-x_{\eta} y_{\xi} .
$$

The scale factors $\eta_{1}$ and $\eta_{2}$ of the transformation are defined as

$$
\eta_{1}=\sqrt{x_{\xi}^{2}+y_{\xi}^{2}}, \quad \eta_{2}=\sqrt{x_{\eta}^{2}+y_{\eta}^{2}} .
$$

Since the mapping is one-to-one, the Jacobian is everywhere non-zero. By the chain rule, we have

$$
\left\{\begin{array}{l}
u_{\xi}=u_{x} x_{\xi}+u_{y} y_{\xi} \\
u_{\eta}=u_{x} x_{\eta}+u_{y} y_{\eta}
\end{array}\right.
$$


which is equivalent to

$$
\left\{\begin{array}{l}
u_{x}=\frac{1}{J}\left(u_{\xi} y_{\eta}-u_{\eta} y_{\xi}\right)=\frac{1}{J}\left(\left(u y_{\eta}\right)_{\xi}-\left(u y_{\xi}\right)_{\eta}\right) \\
u_{y}=\frac{1}{J}\left(u_{\eta} x_{\xi}-u_{\xi} x_{\eta}\right)=\frac{1}{J}\left(\left(u x_{\xi}\right)_{\eta}-\left(u x_{\eta}\right)_{\xi}\right) .
\end{array}\right.
$$

Replacing $u$ with $u_{x}$ and $u_{y}$ in (23) yields

$$
\begin{aligned}
& u_{x x}=\frac{1}{J}\left(\frac{1}{J}\left(u_{\xi} y_{\eta}-u_{\eta} y_{\xi}\right) y_{\eta}\right)_{\xi}-\frac{1}{J}\left(\frac{1}{J}\left(u_{\xi} y_{\eta}-u_{\eta} y_{\xi}\right) y_{\xi}\right)_{\eta} \\
& u_{y y}=\frac{1}{J}\left(\frac{1}{J}\left(u_{\xi} x_{\eta}-u_{\eta} x_{\xi}\right) x_{\eta}\right)_{\xi}-\frac{1}{J}\left(\frac{1}{J}\left(u_{\xi} x_{\eta}-u_{\eta} x_{\xi}\right) x_{\xi}\right)_{\eta} .
\end{aligned}
$$

By adding $u_{x x}$ and $u_{y y}$ and rearranging terms, the first equation in (21) can be written as

$$
\tilde{J} u_{t t}=\tilde{\Delta} u+\tilde{\Delta}_{\sigma} u_{t}, \quad(\xi, \eta) \in \Omega^{\prime},
$$

where we have defined

$$
\begin{gathered}
\tilde{\Delta} u=\left(\alpha_{1} u_{\xi}\right)_{\xi}+\left(\beta u_{\xi}\right)_{\eta}+\left(\beta u_{\eta}\right)_{\xi}+\left(\alpha_{2} u_{\eta}\right)_{\eta}, \\
\tilde{\Delta}_{\sigma} u=\left(\sigma \alpha_{1} u_{\xi}\right)_{\xi}+\left(\sigma \beta u_{\xi}\right)_{\eta}+\left(\sigma \beta u_{\eta}\right)_{\xi}+\left(\sigma \alpha_{2} u_{\eta}\right)_{\eta}, \\
\alpha_{1}=\frac{1}{J}\left(x_{\eta}^{2}+y_{\eta}^{2}\right), \beta=-\frac{1}{J}\left(x_{\eta} x_{\xi}+y_{\eta} y_{\xi}\right), \alpha_{2}=\frac{1}{J}\left(x_{\xi}^{2}+y_{\xi}^{2}\right),
\end{gathered}
$$

and

$$
\tilde{J}=\frac{J}{b}
$$

Using equation (23) to transform $\nabla u \cdot \mathbf{n}$ in the second equation in (21) yields the transformed boundary conditions:

$$
\begin{cases}\gamma_{1} \eta_{2} u-\gamma_{2}\left(\alpha_{1} u_{\xi}+\beta u_{\eta}\right)+\gamma_{3} \eta_{2} u_{t}=0, & (\xi, \eta) \in W \\ \gamma_{1} \eta_{2} u+\gamma_{2}\left(\alpha_{1} u_{\xi}+\beta u_{\eta}\right)+\gamma_{3} \eta_{2} u_{t}=0, & (\xi, \eta) \in E \\ \gamma_{1} \eta_{1} u-\gamma_{2}\left(\alpha_{2} u_{\eta}+\beta u_{\xi}\right)+\gamma_{3} \eta_{1} u_{t}=0, & (\xi, \eta) \in S \\ \gamma_{1} \eta_{1} u+\gamma_{2}\left(\alpha_{2} u_{\eta}+\beta u_{\xi}\right)+\gamma_{3} \eta_{1} u_{t}=0, & (\xi, \eta) \in N .\end{cases}
$$

The complete transformed problem is given by (25), (26) and the initial conditions stated in (21). Applying the energy method (here assuming that also the time derivative of the boundary condition (26) holds) leads to

$$
\begin{aligned}
\frac{d}{d t} E= & -\int_{W} \frac{\gamma_{3}+\sigma \gamma_{1}}{\gamma_{2}} \eta_{2} u_{t}^{2} d \eta-\int_{E} \frac{\gamma_{3}+\sigma \gamma_{1}}{\gamma_{2}} \eta_{2} u_{t}^{2} d \eta \\
& -\int_{N} \frac{\gamma_{3}+\sigma \gamma_{1}}{\gamma_{2}} \eta_{1} u_{t}^{2} d \xi-\int_{S} \frac{\gamma_{3}+\sigma \gamma_{1}}{\gamma_{2}} \eta_{1} u_{t}^{2} d \xi \\
& -\int_{\Omega^{\prime}}\left[\begin{array}{l}
u_{t \xi} \\
u_{t \eta}
\end{array}\right]^{T}\left[\begin{array}{cc}
\sigma \alpha_{1} & \sigma \beta \\
\sigma \beta & \sigma \alpha_{2}
\end{array}\right]\left[\begin{array}{l}
u_{t \xi} \\
u_{t \eta}
\end{array}\right] d \Omega^{\prime},
\end{aligned}
$$


where

$$
E=\frac{1}{2}\left(\int_{\Omega^{\prime}} \tilde{J} u_{t}^{2} d \Omega^{\prime}+\int_{\Omega^{\prime}}\left[\begin{array}{l}
u_{\xi} \\
u_{\eta}
\end{array}\right]^{T}\left[\begin{array}{cc}
\alpha_{1} & \beta \\
\beta & \alpha_{2}
\end{array}\right]\left[\begin{array}{l}
u_{\xi} \\
u_{\eta}
\end{array}\right] d \Omega^{\prime}+B T\right)
$$

and

$$
\begin{aligned}
& B T=\int_{W} \frac{\gamma_{1}}{\gamma_{2}} \eta_{2} u^{2} d \eta+\int_{E} \frac{\gamma_{1}}{\gamma_{2}} \eta_{2} u^{2} d \eta+\int_{N} \frac{\gamma_{1}}{\gamma_{2}} \eta_{1} u^{2} d \xi+\int_{S} \frac{\gamma_{1}}{\gamma_{2}} \eta_{1} u^{2} d \xi \\
& +\int_{W} \sigma \frac{\gamma_{3}}{\gamma_{2}} \eta_{2} u_{t}^{2} d \eta+\int_{E} \sigma \frac{\gamma_{3}}{\gamma_{2}} \eta_{2} u_{t}^{2} d \eta+\int_{N} \sigma \frac{\gamma_{3}}{\gamma_{2}} \eta_{1} u_{t}^{2} d \xi+\int_{S} \sigma \frac{\gamma_{3}}{\gamma_{2}} \eta_{1} u_{t}^{2} d \xi
\end{aligned}
$$

The matrix $\left[\begin{array}{cc}\alpha_{1} & \beta \\ \beta & \alpha_{2}\end{array}\right]$ is positive definite since $\alpha_{1}>0$ and $\alpha_{1} \alpha_{2}-\beta^{2}=$ $\left(x_{\xi} y_{\eta}-x_{\eta} y_{\xi}\right)^{2}=J^{2}>0$. Thus, the problem has an energy estimate if the relations

$$
\frac{\gamma_{1}}{\gamma_{2}} \geq 0, \quad \frac{\gamma_{3}}{\gamma_{2}} \geq 0
$$

hold. The last term in (27) implies damping of the energy for $\sigma>0$.

\subsection{The semi-discrete problem}

In the semi-discrete setting we use the following notation for the matrices corresponding to the continuous variable coefficients, for readability purposes: If $\lambda$ denotes a variable coefficient in the continuous setting, we here denote the matrix with the values of $\lambda(\xi, \eta)$ at the grid points injected on the diagonal by $\lambda$. There is no risk of confusion since it will always be clear from context whether we are in a continuous or semi-discrete setting.

The semi-discrete version of (26) is given by

$$
\left\{\begin{array}{l}
L^{W} v=R_{W}\left\{\gamma_{1} \eta_{2} v-\gamma_{2}\left(\alpha_{1} S_{\xi} v+\beta D_{1 \eta} v\right)+\gamma_{3} \eta_{2} v_{t}\right\}=0 \\
L^{E} v=R_{E}\left\{\gamma_{1} \eta_{2} v+\gamma_{2}\left(\alpha_{1} S_{\xi} v+\beta D_{1 \eta} v\right)+\gamma_{3} \eta_{2} v_{t}\right\}=0 \\
L^{S} v=R_{S}\left\{\gamma_{1} \eta_{1} v-\gamma_{2}\left(\alpha_{2} S_{\eta} v+\beta D_{1 \xi} v\right)+\gamma_{3} \eta_{1} v_{t}\right\}=0 \\
L^{N} v=R_{N}\left\{\gamma_{1} \eta_{1} v+\gamma_{2}\left(\alpha_{2} S_{\eta} v+\beta D_{1 \xi} v\right)+\gamma_{3} \eta_{1} v_{t}\right\}=0
\end{array}\right.
$$

In the numerical scheme we also impose the time-derivative of the boundary conditions when $\sigma>0$. For instance, if we have the boundary condition $L v=f$, we impose both $L v=f$ and $L v_{t}=f_{t}$ using the SAT technique.

The semi-discrete approximation of (25) and (26) using the SBP-SAT method is

$$
\begin{aligned}
\tilde{J} v_{t t} & =D_{2 \xi}^{\left(\alpha_{1}\right)} v+D_{1 \xi} \beta D_{1 \eta} v+D_{1 \eta} \beta D_{1 \xi} v+D_{2 \eta}^{\left(\alpha_{2}\right)} v \\
& +D_{2 \xi}^{\left(\sigma \alpha_{1}\right)} v_{t}+D_{1 \xi} \sigma \beta D_{1 \eta} v_{t}+D_{1 \eta} \sigma \beta D_{1 \xi} v_{t}+D_{2 \eta}^{\left(\sigma \alpha_{2}\right)} v_{t} \\
& +\tau_{1} H_{\xi}^{-1} L^{W} v+\tau_{1} H_{\xi}^{-1} L^{E} v+\tau_{2} H_{\eta}^{-1} L^{S} v+\tau_{2} H_{\eta}^{-1} L^{N} v \\
& +\tau_{3} \sigma H_{\xi}^{-1} L^{W} v_{t}+\tau_{3} \sigma H_{\xi}^{-1} L^{E} v_{t}+\tau_{4} \sigma H_{\eta}^{-1} L^{S} v_{t}+\tau_{4} \sigma H_{\eta}^{-1} L^{N} v_{t} .
\end{aligned}
$$


The first main result of the present study is stated in the following theorem:

Theorem 3.1 The scheme (32) is stable if $\tau_{1}=\tau_{2}=\tau_{3}=\tau_{4}-\frac{1}{\gamma_{2}}$ and (30) holds.

Proof Applying the energy method by multiplying (32) by $v_{t}^{T} H_{\xi} H_{\eta}$ and adding the transpose leads to

$$
\begin{aligned}
\frac{d}{d t} E_{H}= & v_{t}^{T}\left(1+\tau_{1} \gamma_{2}\right) H_{\eta} \alpha_{1}\left(-R_{W}+R_{E}\right) S_{\xi} v+ \\
& v_{t}^{T}\left(1+\tau_{2} \gamma_{2}\right) H_{\xi} \alpha_{2}\left(-R_{S}+R_{N}\right) S_{\eta} v+ \\
& v_{t}^{T}\left(1+\tau_{3} \gamma_{2}\right) H_{\eta} \sigma \alpha_{1}\left(-R_{W}+R_{E}\right) S_{\xi} v_{t}+ \\
& v_{t}^{T}\left(1+\tau_{4} \gamma_{2}\right) H_{\xi} \sigma \alpha_{2}\left(-R_{S}+R_{N}\right) S_{\eta} v_{t}+ \\
& v_{t}^{T}\left(1+\tau_{1} \gamma_{2}\right) H_{\eta}\left(-R_{W}+R_{E}\right) \beta D_{1 \eta} v \quad+ \\
& v_{t}^{T}\left(1+\tau_{2} \gamma_{2}\right) H_{\xi}\left(-R_{S}+R_{N}\right) \beta D_{1 \xi} v \quad+ \\
& v_{t}^{T}\left(1+\tau_{3} \gamma_{2}\right) H_{\eta}\left(-R_{W}+R_{E}\right) \sigma \beta D_{1 \eta} v_{t}+ \\
& v_{t}^{T}\left(1+\tau_{4} \gamma_{2}\right) H_{\xi}\left(-R_{S}+R_{N}\right) \sigma \beta D_{1 \xi} v_{t}+ \\
& v_{t}^{T}\left(\tau_{1} \gamma_{3}+\tau_{3} \sigma \gamma_{1}\right) H_{\eta} \eta_{2}\left(R_{W}+R_{E}\right) v_{t}+ \\
& v_{t}^{T}\left(\tau_{2} \gamma_{3}+\tau_{4} \sigma \gamma_{1}\right) H_{\xi} \eta_{1}\left(R_{S}+R_{N}\right) v_{t}+ \\
& -v_{t}^{T} H_{\eta} M_{\xi}^{\left(\sigma \alpha_{1}\right)} v_{t}-v_{t}^{T} H_{\xi} M_{\eta}^{\left(\sigma \alpha_{2}\right)} v_{t}+ \\
& -2\left(D_{1 \xi} v_{t}\right)^{T} \sigma \beta H_{\xi} H_{\eta}\left(D_{1 \eta} v_{t}\right),
\end{aligned}
$$

where

$$
\begin{aligned}
E_{H}= & \frac{1}{2} v_{t}^{T} H_{\xi} H_{\eta} \tilde{J} v_{t}+ \\
& \frac{1}{2}\left(v^{T} H_{\eta} M_{\xi}^{\left(\alpha_{1}\right)} v+v^{T} H_{\xi} M_{\eta}^{\left(\alpha_{2}\right)} v+2\left(D_{1 \xi} v\right)^{T} \beta H_{\xi} H_{\eta}\left(D_{1 \eta} v\right)\right)+ \\
& \frac{1}{2}\left(-v^{T} \tau_{1} \gamma_{1} H_{\eta} \eta_{2}\left(R_{W}+R_{E}\right) v-v^{T} \tau_{2} \gamma_{1} H_{\xi} \eta_{1}\left(R_{S}+R_{N}\right) v\right)+ \\
& \frac{1}{2}\left(-v_{t}^{T} \tau_{3} \sigma \gamma_{3} H_{\eta} \eta_{2}\left(R_{W}+R_{E}\right) v_{t}-v_{t}^{T} \tau_{4} \sigma \gamma_{3} H_{\xi} \eta_{1}\left(R_{S}+R_{N}\right) v_{t}\right) .
\end{aligned}
$$

By choosing $\tau_{1}=\tau_{2}=\tau_{3}=\tau_{4}=-\frac{1}{\gamma_{2}}$ we obtain an energy estimate completely analogous to (27). If (30) holds, we have a non-growing energy.

\section{Model problem}

\subsection{The continuous problem}

We consider sound propagation over the terrain shown in Figure 2. A source emitting spherical waves with a frequency of $50 \mathrm{~Hz}$ is placed at range $r=0$, height $z=10 \mathrm{~m}$. 


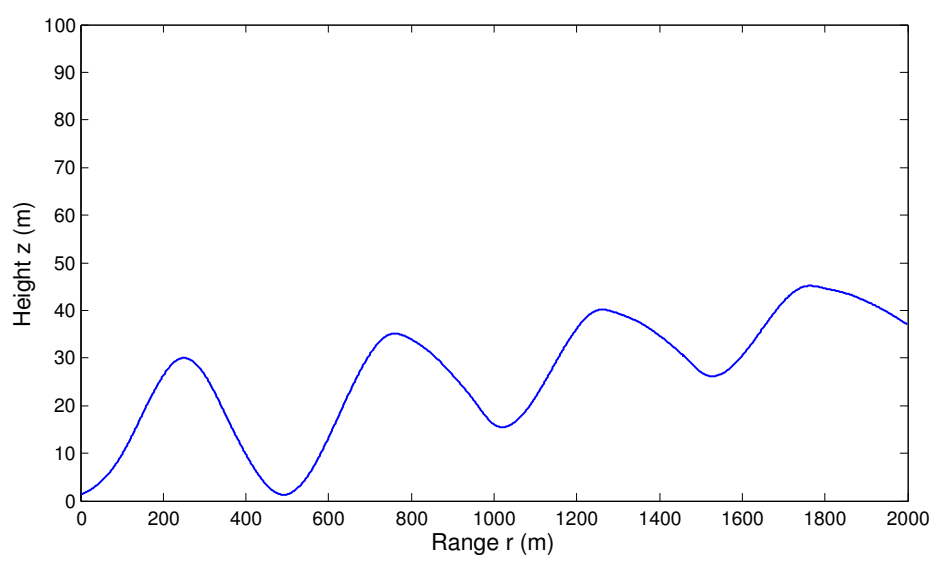

Figure 2: The topography

The propagation of sound waves is governed by the acoustic wave equation

$$
u_{t t}=b \Delta u,
$$

where $u$ is the acoustic pressure and $b$ is the square of the wave velocity. As in Section 3.2, we introduce dissipation,

$$
u_{t t}=b \Delta u+b \nabla \cdot\left(\sigma \nabla u_{t}\right) .
$$

Expressing equation (34) in cylindrical coordinates $(r, \phi, z)$ and assuming symmetry in the azimuthal direction (the $\phi$-direction) results in the axisymmetric two-dimensional restriction of (34),

$$
\frac{1}{b} u_{t t}=\frac{1}{r}\left(r u_{r}\right)_{r}+u_{z z}+\frac{1}{r}\left(\sigma r u_{r}\right)_{r}+\left(\sigma u_{z}\right)_{z} .
$$

We construct the physical domain by introducing artificial boundaries in the manner shown in Figure 3. In the simulations, the west boundary was placed at $r=1 \mathrm{~m}$ and the north boundary at heights ranging from $z=300$ $\mathrm{m}$ to $z=750 \mathrm{~m}$. The dot at the $z$-axis marks the location of the source, just outside the west boundary of the domain.

The boundary condition at the south boundary is a locally-reacting impedance condition given by (see [40])

$$
\left(\frac{p \omega_{0}}{c}-\frac{\chi}{2}\right) u+\nabla u \cdot \mathbf{n}+\frac{q}{c} u_{t}=0,
$$

where $c$ is the wave speed, $\mathbf{n}$ is the unit outward normal, $\omega_{0}$ is the angular frequency of the source, $\chi$ is the curvature and $p$ and $q$ are real numbers that satisfy the relation

$$
p+q i=\frac{i}{\hat{Z}},
$$




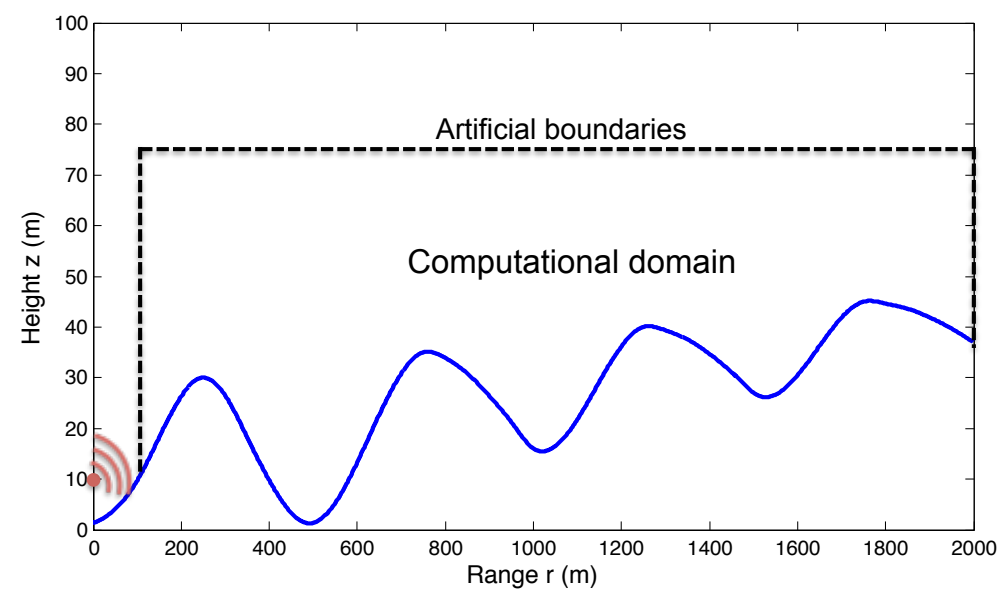

Figure 3: Qualitative description of the physical domain. The dot at the $z$-axis represents the point source

where $\hat{Z}$ is the normalized sound impedance, $\hat{Z}=18.3+17.5 i$. The curvature $\chi$ is defined as

$$
\chi(r)=\frac{-H_{r r}(r)}{\left(1+H_{r}^{2}(r)\right)^{3 / 2}},
$$

where $H(r)$ denotes the height of the ground at horizontal position $r$.

The north and east boundaries are artificial boundaries that are introduced to truncate the unbounded domain. We need to avoid reflections at these boundaries. Absorption of waves at artificial boundaries is an important numerical issue. One possible approach is to apply an absorbing boundary condition (ABC), for example the first order Engquist Majda ABC [9],

$$
\left\{\begin{array}{l}
\left.\left(u_{t}+\sqrt{b} \nabla u \cdot \mathbf{n}\right)\right|_{z=z_{\max }}=0, \\
\left.\left(u_{t}+\sqrt{b} \nabla u \cdot \mathbf{n}+\frac{\sqrt{b}}{2 r} u\right)\right|_{r=r_{\max }}=0 .
\end{array}\right.
$$

However, a first order Engquist Majda ABC is perfectly absorbing only at normal incidence. At $45^{\circ}$ incidence on a straight boundary, $17 \%$ of the incoming wave is reflected, and close to glancing the reflection coefficient tends to unity. Another, more efficient approach is to introduce an absorbing layer (AL) close to the artificial boundary. This can be done by smoothly increasing the dissipation coefficient $\sigma$ from zero to some fixed value. The dissipation must increase quickly enough that the waves are damped efficiently inside the layer, but it must also increase smoothly enough that we avoid reflections at the interface between the $\mathrm{AL}$ and the interior domain. The wider one can afford to make the layer, the easier it is to find a function $\sigma$ that fulfills both criteria. This AL is a special case of the method presented by Appelö and Colonius in [3]. Appelö and Colonius also slowed 
down waves inside the layer by stretching the grid at the boundaries and included higher-order dissipation operators for better performance. Since the focus in the present study is not on optimal absorbing layers, we here settle for a simple version of their AL. We will verify that the truncation of the domain does not affect the solution by placing the artificial boundaries at different locations in the simulations.

At the west boundary, the boundary condition is determined by the source. Consider a point source with amplitude $A$ and frequency $f$. At a distance $\tilde{r}$ from the source, the acoustic pressure is given by

$$
u^{(s)}(\tilde{r}, t)=\frac{A}{\tilde{r}} \sin \left(2 \pi f\left(t-\frac{\tilde{r}}{c}\right)\right) .
$$

Now let the source be located at $(r, z)=\left(0, z_{0}\right)$. The distance $\tilde{r}$ from the source to a point $(r, z)$ is given by

$$
\tilde{r}=\sqrt{r^{2}+\left(z-z_{0}\right)^{2}} .
$$

Combining (40) and (41) yields

$$
u^{(s)}(r, z, t)=\frac{A}{\sqrt{r^{2}+\left(z-z_{0}\right)^{2}}} \sin \left(2 \pi f\left(t-\frac{\sqrt{r^{2}+\left(z-z_{0}\right)^{2}}}{c}\right)\right) .
$$

We will impose this boundary data on the west boundary. This can be done using Dirichlet boundary condition by extending the SAT technique developed in [31]. However, in the present study we instead impose the data using a mixed boundary condition (which allows for a stronger energy estimate in terms of boundary data),

$$
u_{t}+\sqrt{b} \nabla u \cdot \mathbf{n}+\frac{\sqrt{b}}{2 r} u=u_{t}^{(s)}+\sqrt{b} \nabla u^{(s)} \cdot \mathbf{n}+\frac{\sqrt{b}}{2 r} u^{(s)}, \quad r=r_{\min } .
$$

We now introduce logical coordinates $(\xi, \eta)$ and perform a transformation from the unit square $(0 \leq \xi \leq 1,0 \leq \eta \leq 1)$ onto the physical domain $(r(\xi, \eta), z(\xi, \eta)) \in \Omega$. Equation (35) transforms into

$$
\tilde{J} u_{t t}=\tilde{\Delta} u+\tilde{\Delta}_{\sigma} u_{t}, \quad(\xi, \eta) \in \Omega^{\prime},
$$

where we have defined

$$
\begin{gathered}
\tilde{J}=\frac{r J}{b}, \\
\tilde{\Delta} u=\left(\alpha_{1} u_{\xi}\right)_{\xi}+\left(\beta u_{\xi}\right)_{\eta}+\left(\beta u_{\eta}\right)_{\xi}+\left(\alpha_{2} u_{\eta}\right)_{\eta}, \\
\tilde{\Delta}_{\sigma} u=\left(\sigma \alpha_{1} u_{\xi}\right)_{\xi}+\left(\sigma \beta u_{\xi}\right)_{\eta}+\left(\sigma \beta u_{\eta}\right)_{\xi}+\left(\sigma \alpha_{2} u_{\eta}\right)_{\eta},
\end{gathered}
$$

and

$$
\alpha_{1}=\frac{r}{J}\left(r_{\eta}^{2}+z_{\eta}^{2}\right), \beta=-\frac{r}{J}\left(r_{\xi} r_{\eta}+z_{\xi} z_{\eta}\right), \alpha_{2}=\frac{r}{J}\left(r_{\xi}^{2}+z_{\xi}^{2}\right)
$$


Table 1: Number of grid points corresponding to different resolutions. North boundary at $z=500 \mathrm{~m}$

\begin{tabular}{llll}
\hline Resolution (points per wavelength) & $N_{r}$ & $N_{z}$ & $N_{r} \cdot N_{z}$ \\
\hline 6 & 1801 & 451 & $0.81 \cdot 10^{6}$ \\
9 & 2701 & 676 & $1.83 \cdot 10^{6}$ \\
12 & 3601 & 901 & $3.24 \cdot 10^{6}$ \\
\hline
\end{tabular}

The model that we solve is the equation (44) with the boundary conditions (36) and (43) at the south and west boundaries, and either the ABC (39) or the AL at the north and east boundaries. In the ABC approach, $\sigma$ is identically zero. In the $\mathrm{AL}$ approach, $\sigma$ is non-zero close to the north and east boundaries. The equation (44) has the same form as (25), and all the boundary conditions (36), (43) and (39) are of the mixed type analyzed in Section 3. Thus, the analysis performed in Section 3, proving well-posedness for the continuous problem and stability for the discrete scheme, holds for this model too.

\subsection{Implementation details}

We have implemented a fourth-order SBP-SAT method of the model problem. The spatial discretization is thus fourth-order accurate in the interior scheme and second-order accurate in the boundary closures. For completeness we have included the operators (first presented in [28]) in Appendix. The classical fourth-order accurate Runge-Kutta method was used for discretization in time.

In order to apply the solver to the model problem, a computational grid must be constructed in the physical domain. Generating a good grid on a complex domain is not a trivial task. If the grid is not smooth enough, the convergence rate will decrease. In this case, the terrain profile has only two continuous derivatives, and hence we suspect that the grid will not support high-order accuracy. We have used Pointwise, a commercial software for creating grids. Figure 4 shows an example of a coarse grid generated in Pointwise. Table 1 lists the number of gridpoints required when using 6, 9 and 12 points per acoustic wavelength, with the north boundary at a height of $500 \mathrm{~m}$.

In the simulations, we time-advanced until the solution became periodic in time and then computed the amplitude of the sound waves by measuring $|v|_{\text {max }}$, the maximum absolute value of the solution during one period, one meter above ground. The propagation loss $P$ (measured in $\mathrm{dB}$ ) was computed as

$$
P=-20 \cdot \log \left(\frac{|v|_{\max }}{A}\right)
$$




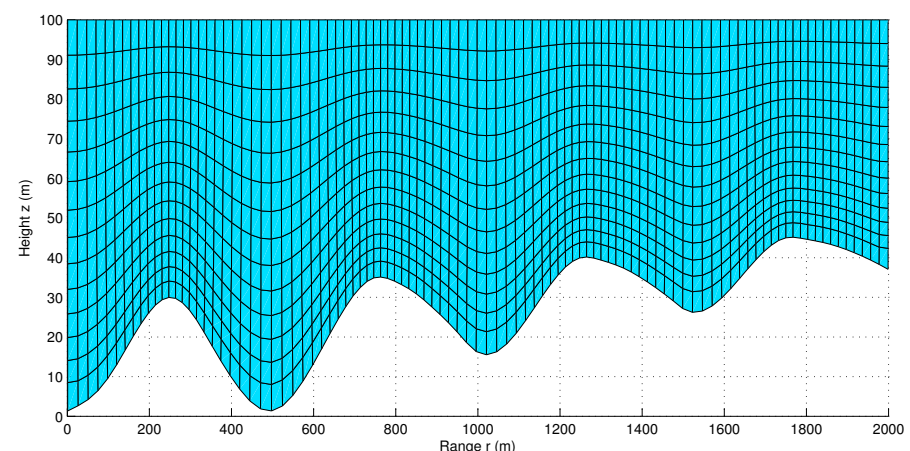

Figure 4: Coarse example grid

where $A$ is the amplitude of the point source.

\section{The parabolic equation method}

The PE method used in the model problem of Section 8 is described briefly below. More details can be found in [20].

As an initial step, a smooth approximation $h(r)$ of the ground height as function of range is computed from the data, using an interpolating or a variance-reducing B-spline expansion [6, Ch. XI], the choice depending on the smoothness of the data. The geometry is then mapped from the physical $(r, z)$ domain to a rectangle in the $(\xi, \eta)$ plane by an orthogonal curvilinear transform

$$
r=r(\xi, \eta), \quad z=z(\xi, \eta), \quad r_{\xi} r_{\eta}+z_{\xi} z_{\eta}=0,
$$

such that $\eta$ is constant along the boundaries of the computational domain. Assuming cylindrical symmetry, the Helmholtz equation for the complex pressure $u(\xi, \eta)$ is

$$
f\left(f^{-1} u_{\xi}\right)_{\xi}+f\left(g^{-1} u_{\eta}\right)_{\eta}+k^{2} a^{2} u=0,
$$

where, using the unit $\mathrm{dB} /$ wavelength for the attenuation $\alpha$,

$$
\begin{aligned}
f & =\rho a / r b, & g & =\rho b / r a, \\
a & =\left(r_{\xi}^{2}+z_{\xi}^{2}\right)^{1 / 2}, & b & =\left(r_{\eta}^{2}+z_{\eta}^{2}\right)^{1 / 2}, \quad k=\frac{\omega}{c}\left(1+i \alpha \frac{\log (10)}{40 \pi}\right) .
\end{aligned}
$$

The PE approximations are derived by writing (47) in the form

$$
T^{2} u=(1-L) u-k_{0}^{-2} R u,
$$


where $k_{0}$ is a reference wavenumber, $T$ and $L$ are differential operators, and $R$ a function:

$$
\begin{aligned}
T u & =-i k_{0}^{-1} f^{1 / 2}\left(f^{-1 / 2} u\right)_{\xi}, \\
L u & =-k_{0}^{-2}\left[f\left(g^{-1}\left(u_{\eta}\right)\right)_{\eta}+k^{2} a^{2} u\right]+u, \\
R & =\left(3 f_{\xi}^{2}-2 f f_{\xi \xi}\right) /\left(4 f^{2}\right) .
\end{aligned}
$$

Discarding the term $R u$, which is small since $R \sim \xi^{-2}$ as $\xi$ grows, equation (48) is simplified to

$$
T^{2} u=(1-L) u \text {. }
$$

The PE schemes compute one-way solutions to (52) by solving

$$
T u=\sqrt{1-L} u
$$

with the pseudo differential operator $\sqrt{1-L}$ replaced by a rational function of $L$,

$$
T u=\frac{P_{m}(L)}{Q_{n}(L)} u,
$$

analoguously with [2]. $P_{m}$ and $Q_{n}$ are polynomials of degrees $m$ and $n$ in the Padé approximation

$$
\sqrt{1-x}=P_{m}(x) / Q_{n}(x)+O\left(x^{m+n+1}\right), \quad x \rightarrow 0 .
$$

Thus the JEPE PE-approximations are

$$
\begin{aligned}
u & =Q_{n}(L) v, \\
T Q_{n}(L) v & =P_{m}(L) v, \quad m=\max (n, 1), \quad n=0,1,2, \ldots
\end{aligned}
$$

Increasing the Padé order $n$ reduces the phase error as function of elevation angle, but also increases the computational work. In practice, Padé orders $n=0,1,2$ are the most frequently used and correspond to the narrow angle $\left(15^{\circ}\right)$, the wide angle $\left(35^{\circ}\right)$ and the very wide angle $\left(55^{\circ}\right)$ approximations, cf. [19, Sec. 6.2.4].

Equation (57) with initial conditions at $\xi=\xi_{0}$ and boundary conditions at $\eta=0$ (the upper boundary) and $\eta=-H$ (the ground) is solved using the method of lines. Thus, $u, T, L$ and the boundary conditions are discretized vertically using a centered second-order finite difference scheme, [42, Sec. 9]. The vertically discretized form of equation (57) is a system of ODEs (omitting the indices $m$ and $n$ )

$$
\frac{d}{d \xi} \mathbf{D} Q(\mathbf{L}) \mathbf{w}=i k_{0} \mathbf{D}[P(\mathbf{L})-Q(\mathbf{L})] \mathbf{w} \quad \mathbf{w}\left(\xi_{0}\right)=\mathbf{w}_{0}
$$

for the scaled and wavenumber-shifted complex pressure

$$
\mathbf{w}(\xi)=e^{-i k_{0}} \xi^{1 / 2} \mathbf{v}(\xi)
$$


$\mathbf{D}$ and $\mathbf{L}$ are diagonal and tri-diagonal matrix-valued functions of $\xi$, respectively, with $\mathbf{D}$ real and the imaginary part of $\mathbf{L}$ diagonal and non-positive. The initial profile $\mathbf{w}_{0}$ is computed from the given source data (height and vertical directivity), by low-pass filtering w.r.t. vertical wave number to the validity interval of the $\mathrm{PE}$ scheme. Equation 58 is then solved by a two-step fourth order A-stable second derivative method by Jeltsch (method J4 in [18]).

\section{The ray interpolation method}

In the ray interpolation method the ground height is described by a smooth function of range identical to that in the PE method described in Section 5. A ray trajectory $(r(s), z(s))$ where $s$ is arc length, is a solution to the ODE system [19, Sec. 3.2.1]

$$
\begin{aligned}
d r / d s & =\cos (\phi) \\
d z / d s & =\sin (\phi) \\
d \phi / d s & =\{\sin (\phi) \partial c / \partial r-\cos (\phi) \partial c / \partial z\} / c
\end{aligned}
$$

$c=c(r, z)$ is the sound speed and $\phi=\phi(s)$ the elevation angle of the ray. In the high-frequency limit, the ray trajectories are streamlines of the acoustic intensity field i.e. propagation paths of acoustic energy. The wavefield at a point $(r, z)$ is then a sum of contributions from all rays passing through $(r, z)$ - all eigenrays from the source to $(r, z))$. Each eigenray contributes to the sum with the field inside an infinitesimal tube surrounding the ray. With a mono-frequency monopole source with amplitude $P_{0}$ at $1 \mathrm{~m}$ range, the value of the ray-tube field along an eigenray $(r(s), z(s))$ is

$$
P(f, s)=P_{0} \alpha(s) e^{i 2 \pi f \tau(s)} e^{i \pi n_{c}(s) / 2} \Pi_{j=1}^{n_{b}(s)} \gamma_{j}
$$

where

$$
\begin{aligned}
f & \text { frequency } \\
\alpha(s)= & \frac{\cos \phi_{0}}{r A(s)} \quad \text { ray tube area factor } \\
\phi_{0}= & \phi(0) \quad \text { launch angle } \\
A(s)= & -\sin (\phi) \partial r / \partial \phi_{0}+\cos (\phi) \partial z / \partial \phi_{0} \\
\tau(s) & \text { travel time along ray } \\
n_{c}(s) & \text { number of caustic points along ray } \\
n_{b}(s) & \text { number of ground reflections along ray } \\
\gamma_{j} & \text { reflection coefficient for } \mathrm{j}^{\prime} \text { th ground reflection }
\end{aligned}
$$

The wavefield $P(f, r)$ at points $(r, h(r)) 0<r<R$ on the ground from a source at $\left(0, z_{s}\right)$ is computed in two steps. 
First, a pre-defined number $K$ of ray paths $\left(r_{j}(s), z_{j}(s)\right)$ are computed by solving the ray ODEs with a fourth order Runge-Kutta method [12, p. 178] with variable stepsize and local error control. The rays start from the source $\left(r_{j}(0), z_{j}(0)\right)=\left(0, z_{s}\right)$ with uniformly distributed vertical launch angles $\Phi_{j}, j=1, \ldots, K$, and the ODE system (60) is augmented with one equation each for the travel time $\tau(s)$ along the ray and the partial derivatives $\partial r(s) / \partial \phi_{0}, \partial z(s) / \partial \phi_{0}, \partial \phi(s) / \partial \phi_{0}$ with respect to launch angle $\phi_{0}$. Rays are reflected at the ground and terminated at the maximal range $r=R$ or at the upper boundary $z=Z$ of the computational region. The number and the locations of caustic points and ground reflections along each ray are determined.

Then, for each point $\left(r_{j}, h\left(r_{j}\right)\right)$ on a receiver grid, the field $P(f, r)$ is obtained as a sum of contributions of the form (61) approximating the eigenrays by cubic interpolation to appropriate ray subsets.

\section{Convergence results}

In this section we verify the implementation of the fourth-order SBP-SAT method and investigate the quality if the grid in a series of convergence studies. We will calculate the convergence rate $q$ as

$$
q=\log _{10}\left(\frac{\left\|v_{r e f}-v^{\left(N_{2}\right)}\right\|_{h}}{\left\|v_{r e f}-v^{\left(N_{1}\right)}\right\|_{h}}\right) / \log _{10}\left(\frac{N_{1}}{N_{2}}\right)^{1 / d},
$$

where $d$ is the dimension ( $d=2$ here), $v_{r e f}$ is a reference solution, $v^{(N)}$ is the corresponding numerical solution with $N$ grid points and $\left\|v_{r e f}-v^{(N)}\right\|_{h}$ is the discrete $l^{2}$ norm of the error.

\subsection{Convergence study without absorbing layer}

To separate the effects of the grid from the numerical method, we here present a convergence study on a smooth curvilinear grid. We use the analytical solution

$$
u^{(a)}(r, z, t)=\frac{A}{\sqrt{r^{2}+\left(z-z_{0}\right)^{2}}} \sin \left(2 \pi f\left(t-\frac{\sqrt{r^{2}+\left(z-z_{0}\right)^{2}}}{c}\right)\right)
$$

which is the pressure field created by a point source with frequency $f$ and amplitude $A$, located at $(r, z)=\left(0, z_{0}\right)$. We here set $A=1, f=12.5$ $\mathrm{Hz}$ and $z_{0}=10 \mathrm{~m}$. In the computations we use the same setup as for the benchmark problem, except that we here set the dissipation coefficient $\sigma$ to zero everywhere (in order to have an analytical solution) and impose the analytical solution $u^{(a)}$ as initial and boundary data. The setup with the smooth domain and the initial condition is shown in Figure 5. The 


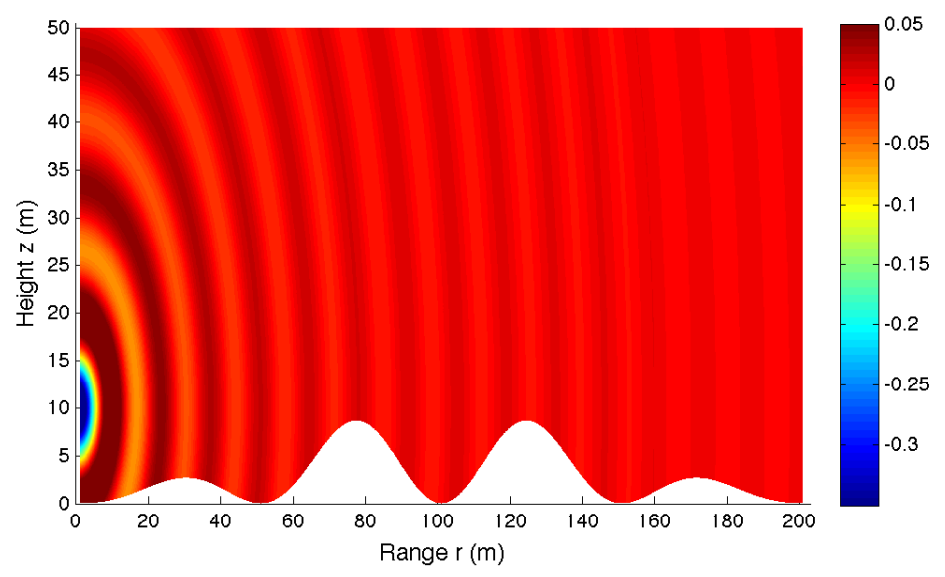

Figure 5: The analytical solution at time $t=0$ on a smooth grid

Table 2: $\log \left(l_{2}-\right.$ errors $)$ and convergence rates on a smooth grid, without absorbing layer

\begin{tabular}{lll}
\hline$N_{r} \times N_{z}$ & $\log e_{l_{2}}$ & $q$ \\
\hline $81 \times 21$ & -0.26 & 0.00 \\
$161 \times 41$ & -1.30 & 3.46 \\
$321 \times 81$ & -2.62 & 4.45 \\
$641 \times 161$ & -3.73 & 3.64 \\
$1281 \times 321$ & -4.88 & 3.80 \\
\hline
\end{tabular}

convergence results are presented in Table 2. We note that we obtain the expected fourth order convergence rate.

Next, we investigate the quality of the grid generated (using the commercial grid-generator Pointwise) for the benchmark problem by running a convergence study with exactly the same setup on that grid. The results are presented in Table 3. We note that we obtain approximately third order convergence on this grid and draw the conclusion that the grid, as expected, is not smooth enough to support high-order accuracy (higher than third order). When solving the benchmark problem, we will thus have to make do with third order convergence.

\subsection{Convergence study with absorbing layer}

To verify also the implementation of the $\mathrm{AL}$, we here perform a convergence study with a non-zero dissipation coefficient $\sigma$ on the benchmark grid. The solution obtained with $3601 \times 881$ grid points was used as a reference solution. The results are presented in Table 4 . Similar to Table 3, we obtain slightly less than third order convergence on this grid. 
Table 3: $\log \left(l_{2}-\right.$ errors $)$ and convergence rates on the grid generated for the benchmark problem, without absorbing layer

\begin{tabular}{lll}
\hline$N_{r} \times N_{z}$ & $\log e_{l_{2}}$ & $q$ \\
\hline $226 \times 56$ & 0.33 & 0.00 \\
$451 \times 111$ & -0.53 & 2.87 \\
$901 \times 221$ & -1.37 & 2.77 \\
$1801 \times 441$ & -2.19 & 2.72 \\
\hline
\end{tabular}

Table 4: $\log \left(l_{2}-\right.$ errors $)$ and convergence rates on the grid generated for the benchmark problem, with absorbing layer

\begin{tabular}{lll}
\hline$N_{r} \times N_{z}$ & $\log e_{l_{2}}$ & $q$ \\
\hline $226 \times 56$ & 0.46 & 0.00 \\
$451 \times 111$ & -0.32 & 2.59 \\
$901 \times 221$ & -1.09 & 2.56 \\
$1801 \times 441$ & -1.84 & 2.48 \\
\hline
\end{tabular}

\section{Computations}

We have solved the benchmark problem described in Section 4 for two different sound speed profiles:

- profile 1: Constant profile, $c=340 \mathrm{~m} / \mathrm{s}$

- profile 2: Linear profile, $c=c_{0}+k z$ with $c_{0}=340 \mathrm{~m} / \mathrm{s}$ and $k=0.1$ $s^{-1}$.

To guarantee a correct solution it is important to verify: 1 ) that the SPL at ground level is grid-converged, and 2) that reflections at artificial boundaries are negligible. We begin this section by investigating the effects of the artificial boundary treatment, and then perform a grid-convergence study. The grid-converged results obtained with the SBP-SAT method are then compared with the results obtained with the $\mathrm{PE}$ and ray tracing methods.

\subsection{Domain truncation}

To investigate the effect of the artificial boundaries, we computed the propagation loss for different locations of the north boundary, for case 1. In Figure 6 we compare the first order Engquist Majda ABC with the AL approach to truncate the domain at the north boundary. The effects of the reflections using the first order Engquist Majda ABC decrease as we move the north boundary higher, but even with the north boundary at a height of $2000 \mathrm{~m}$ the reflections interfere with the interior waves and cause rapid oscillations 
in the SPL at ground level. The spurious reflections when using the AL are much smaller. After $500 \mathrm{~m}$, the results do not change visibly. In the remaining computations we place the north boundary at $z=500 \mathrm{~m}$ and employ the AL approach to truncate the domain.

Remark We also extended the domain and moved the east boundary further to the right, but the location of the east boundary turned out to have no impact on the SPL at ground level.

\subsection{Grid-convergence}

To verify that the discretization errors are negligible, we study how the computed propagation loss varies with grid refinement. The results are shown in Figure 7 . The curve obtained using 4.5 grid points per wavelength deviates significantly from the others, while the curves corresponding to 6 and 12 grid points per wavelength are almost identical, i.e., indicating grid convergence. In the remaining simulations, grids with 12 points per wavelength were used.

\subsection{Comparison of models}

Figure 8 shows the propagation loss, obtained with the SBP-SAT method, in the entire domain up to a height of $100 \mathrm{~m}$. The effects of the refraction that occurs with profile 2 is most apparent far away from the source.

In Figure 9 we compare the result obtained with the SBP-SAT method with the result published in [40] and the result obtained with the PE method described in Section 5, for profile 1. We observe that the results are in fairly good agreement. The maximum difference between the SBP-SAT and the PE methods in Section 5 and in [40] are $8 \mathrm{~dB}$ and $4 \mathrm{~dB}$, respectively.

In Figure 10 we compare the ray tracing methods with the SBP-SAT method, for profile 1 . The difference between the computed SPL using the SBP-SAT method and the most accurate ray tracing method is always greater than $15 \mathrm{~dB}$ beyond 1100 meters. We also note that the results obtained with the different ray tracing methods differ significantly from one another, and they all under-predict the SPL.

In Figure 11 we compare the SBP-SAT method with the PE and ray tracing methods, with profile 2. The $\mathrm{PE}$ method again shows reasonable agreement with the SBP-SAT method. The ray tracing methods are here in better agreement with the SBP-SAT method than they were in Figure 10 (profile 1), but again they under-predict the SPL, except "Ray interpolation" that now over-predicts (except at the very end of the domain).

\section{Conclusion}

The theory surrounding the SBP-SAT technique has been extended with a result that proves the stability of the SBP-SAT method for the second order 


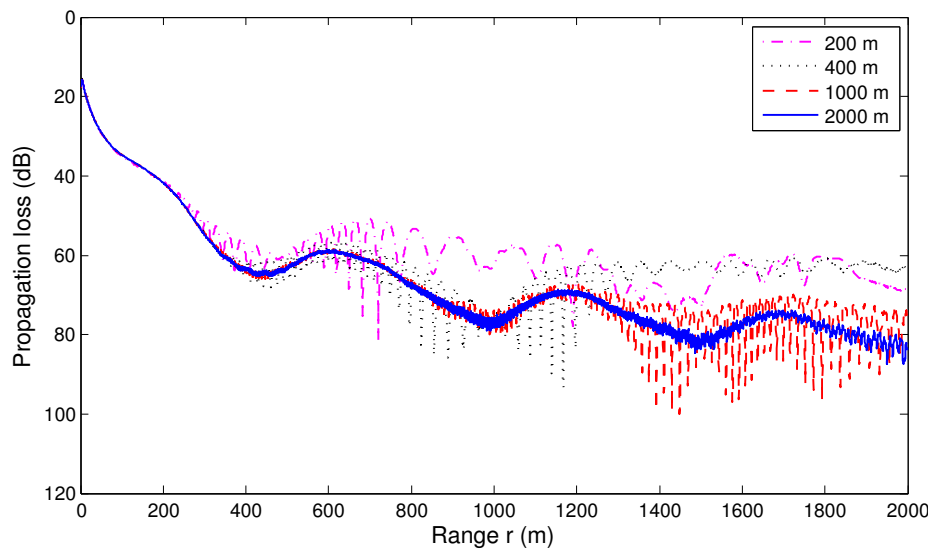

(a) Engquist Majda ABC

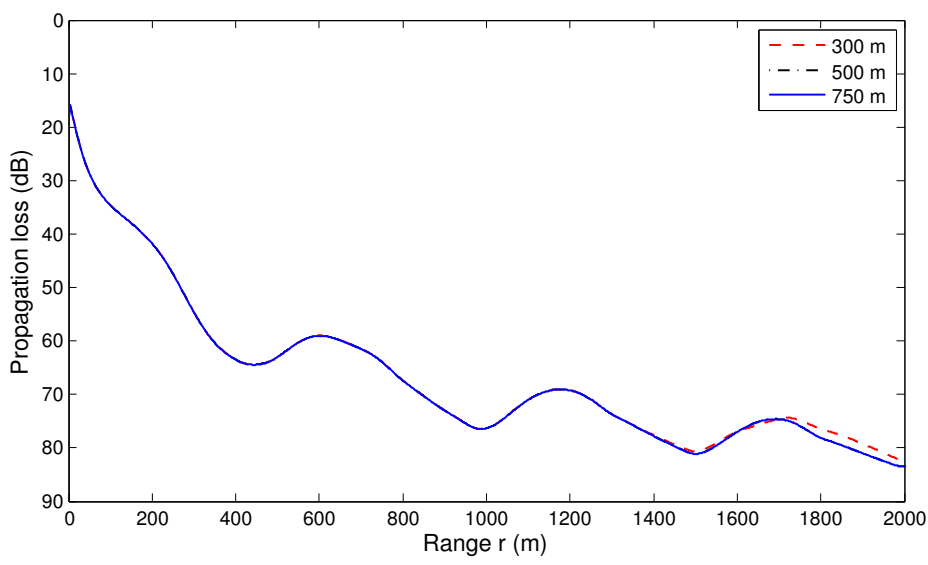

(b) $\mathrm{AL}$

Figure 6: Propagation loss measured $1 \mathrm{~m}$ above ground for different locations of the north boundary, using (a) first order Engquist Majda ABC and (b) an $\mathrm{AL}$ 


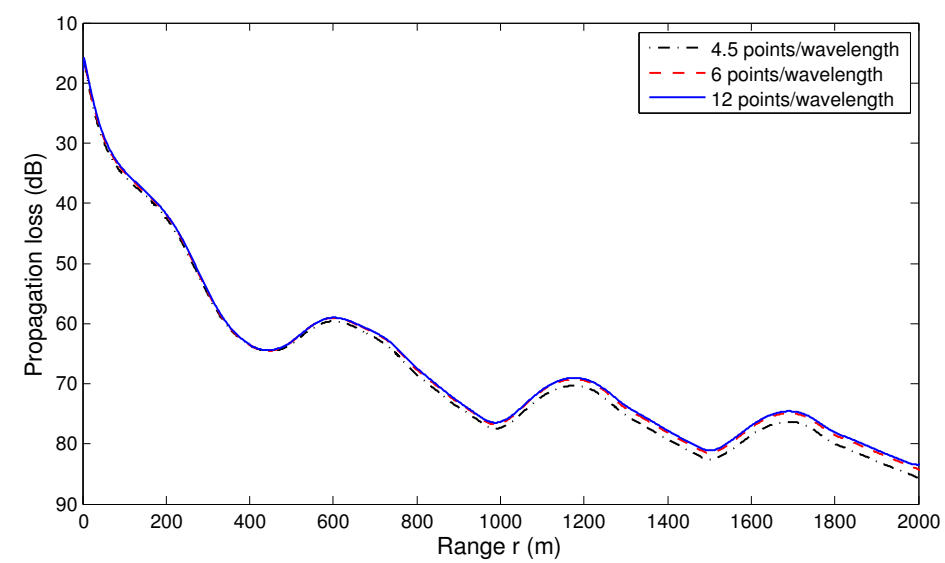

Figure 7: Convergence study. The graphs show the propagation loss $1 \mathrm{~m}$ above the ground for different levels of grid refinement.

wave equation on a curvilinear 2-D domain with mixed boundary conditions. A fourth-order accurate SBP-SAT method has been applied to the benchmark problem on atmospheric sound propagation introduced in [40]. Since the SBP-SAT method is here applied to the full wave equation model, it can be used as a reference against which simpler (and computationally cheaper) methods can be validated.

The present study has shown that, when applying the SBP-SAT method to sound propagation problems, the following should be considered:

- The introduction of artificial boundaries must not affect the solution. One way to achieve this is with carefully constructed absorbing layers.

- A grid generated for a realistic topography might not support highorder accuracy.

- The SPL must be grid-converged.

The results presented in Figures 10 and 11 show that ray tracing methods are not reliable for prediction of SPL in the case of irregular terrain. The PE methods show reasonable agreement with the SBP-SAT method, both with constant speed of sound and with a linear sound speed profile, which is expected since the topography in this problem is rather gentle. One would expect the PE methods, and the ray tracing methods in particular, to be more unreliable in the case of more pronounced topography. This is something we hope to address in a coming study. 


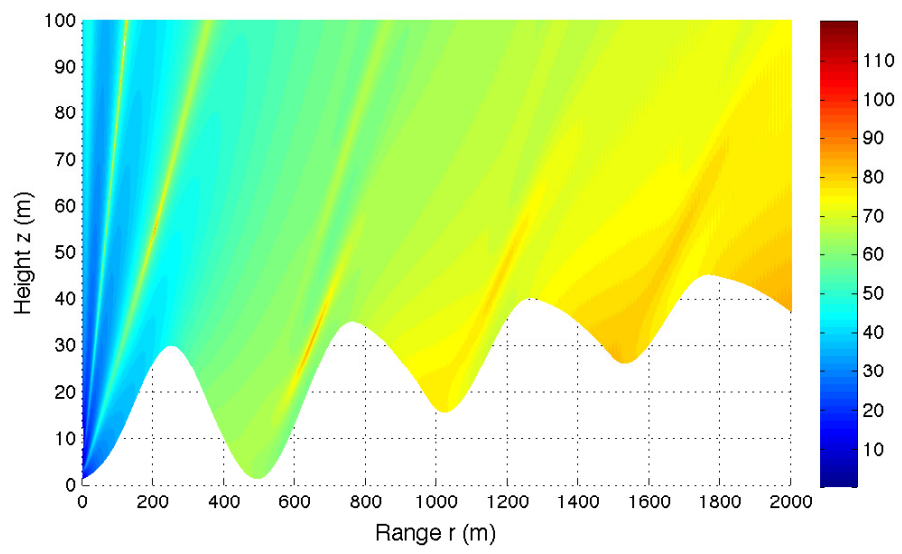

(a) Profile 1

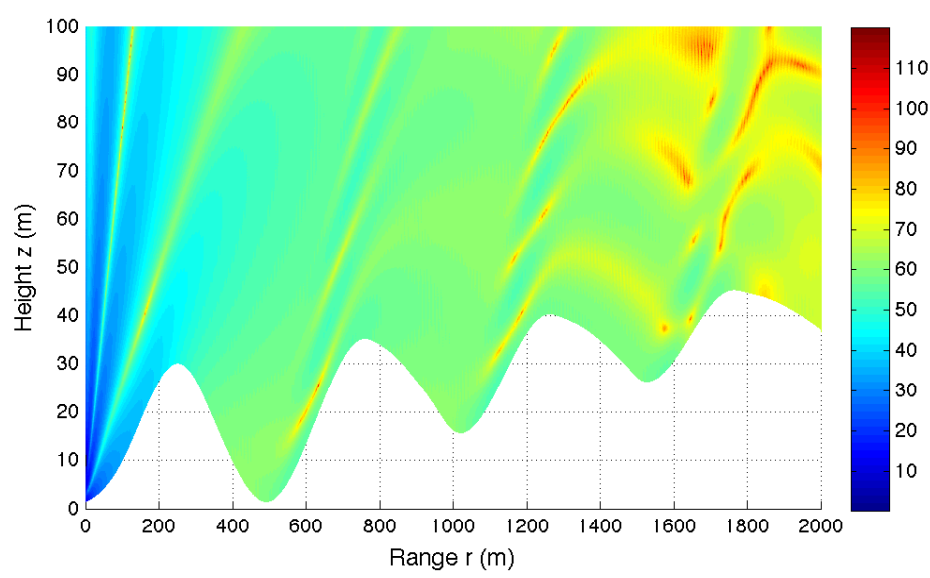

(b) Profile 2

Figure 8: Propagation loss (dB) for different sound speed profiles, (a) profile 1 and (b) profile 2 


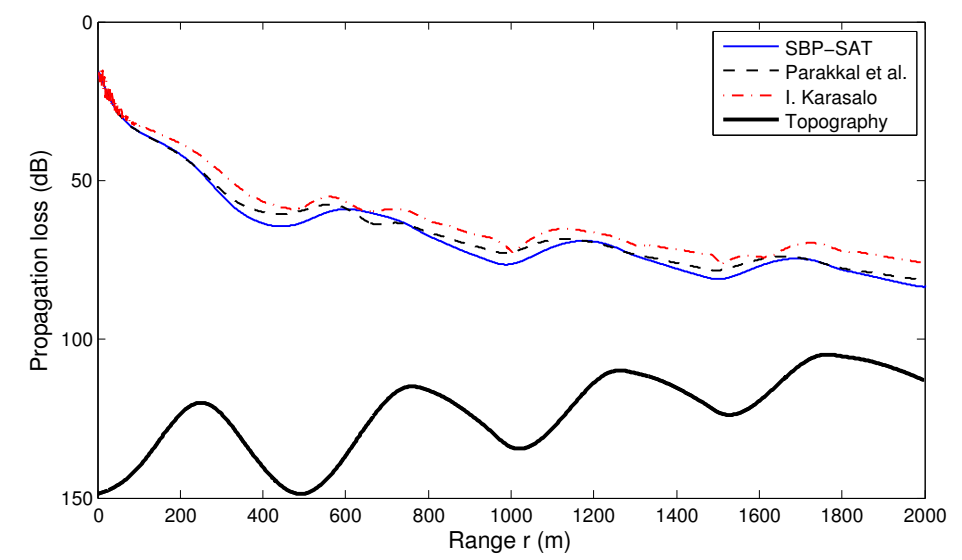

Figure 9: Propagation loss $1 \mathrm{~m}$ above ground for SBP-SAT and PE, with profile 1

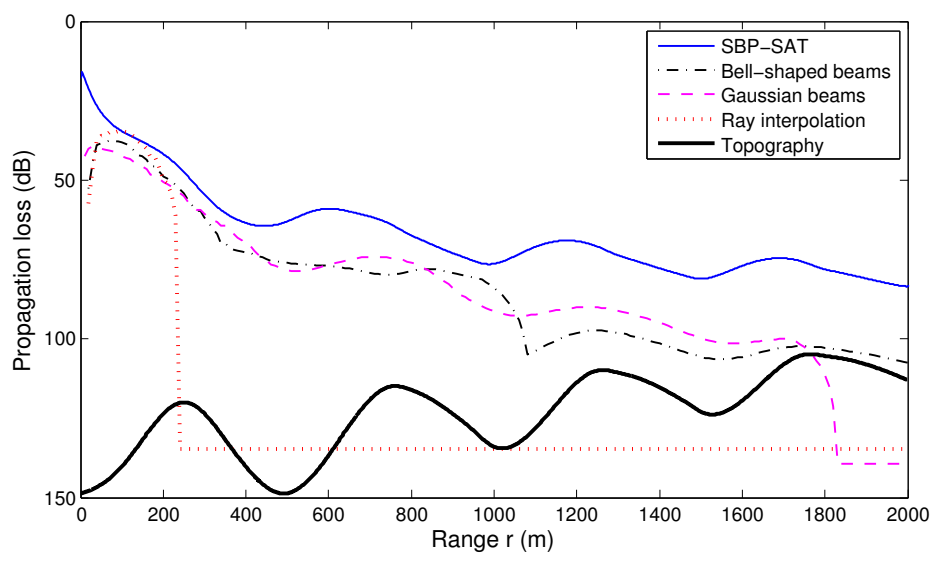

Figure 10: Propagation loss $1 \mathrm{~m}$ above ground for the SBP-SAT method and the ray tracing methods, with profile 1 


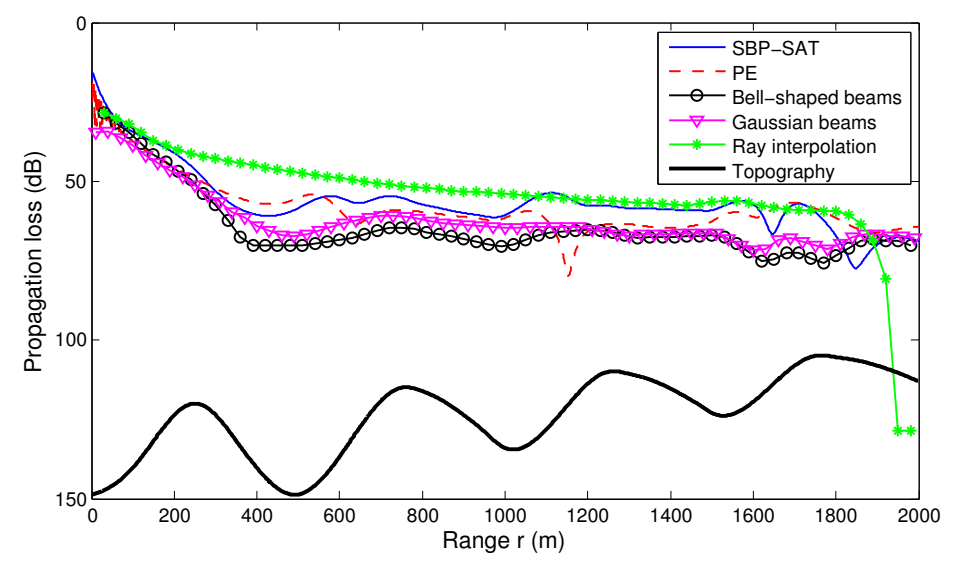

Figure 11: Propagation loss $1 \mathrm{~m}$ above ground for the SBP-SAT method, the $\mathrm{PE}$ and ray tracing methods, with profile 2

\section{A Finite difference operators}

For completeness we present the fourth order SBP operators. Here $h$ denotes the grid-spacing. The interior stencils (in $D_{1}$ and $M^{(b)}$ ) are the standard central 4th order accurate finite difference stencils. At the boundaries we use one-sided stencils that are formally second order accurate. The discrete norm $H$ is defined:

$$
H=h\left[\begin{array}{cccccc}
\frac{17}{48} & & & & & \\
& \frac{59}{48} & & & & \\
& & \frac{43}{48} & & & \\
& & & \frac{49}{48} & & \\
& & & & 1 & \\
& & & & & \ddots
\end{array}\right]
$$

The first derivative SBP operator is given by,

$$
D_{1}=\frac{1}{h}\left[\begin{array}{cccccccc}
-\frac{24}{17} & \frac{59}{34} & -\frac{4}{17} & -\frac{3}{34} & 0 & 0 & 0 & \\
-\frac{1}{2} & 0 & \frac{1}{2} & 0 & 0 & 0 & 0 & \\
\frac{4}{43} & -\frac{59}{86} & 0 & \frac{59}{86} & -\frac{4}{43} & 0 & 0 & \\
\frac{3}{98} & 0 & -\frac{59}{98} & 0 & \frac{32}{49} & -\frac{4}{49} & 0 & \\
0 & 0 & \frac{1}{12} & -\frac{2}{3} & 0 & \frac{2}{3} & -\frac{1}{12} & \\
& & & \ddots & \ddots & \ddots & \ddots & \ddots
\end{array}\right]
$$

The third-order accurate boundary derivative operator $S_{0}$ is given by,

$$
S_{0}=\frac{1}{h}\left[\begin{array}{lllllll}
\frac{11}{6} & -3 & \frac{3}{2} & -\frac{1}{3} & 0 & 0 & \ldots
\end{array}\right]
$$


The interior stencil of $-h M^{(b)}$ at row $i$ is given by $(i=7 \ldots N-6)$ :

$$
\begin{aligned}
& m_{i, i-2}=\frac{1}{6} b_{i-1}-\frac{1}{8} b_{i-2}-\frac{1}{8} b_{i} \\
& m_{i, i-1}=\frac{1}{6} b_{i-2}+\frac{1}{6} b_{i+1}+\frac{1}{2} b_{i-1}+\frac{1}{2} b_{i} \\
& m_{i, i}=-\frac{1}{24} b_{i-2}-\frac{5}{6} b_{i-1}-\frac{5}{6} b_{i+1}-\frac{1}{24} b_{i+2}-\frac{3}{4} b_{i} . \\
& m_{i, i+1}=\frac{1}{6} b_{i-1}+\frac{1}{6} b_{i+2}+\frac{1}{2} b_{i}+\frac{1}{2} b_{i+1} \\
& m_{i, i+2}=\frac{1}{6} b_{i+1}-\frac{1}{8} b_{i}-\frac{1}{8} b_{i+2}
\end{aligned}
$$

The left boundary closure of $-h M^{(b)}$ (given by a $6 \times 6$ matrix) is given by

$$
\begin{aligned}
& m_{1,1}=\frac{12}{17} b_{1}+\frac{59}{192} b_{2}+\frac{27010400129}{345067064608} b_{3}+\frac{69462376031}{2070402387648} b_{4} \\
& m_{1,2}=-\frac{59}{68} b_{1}-\frac{6025413881}{21126554976} b_{3}-\frac{537416663}{7042184992} b_{4} \\
& m_{1,3}=\frac{2}{17} b_{1}-\frac{59}{192} b_{2}+\frac{213318005}{16049630912} b_{4}+\frac{2083938599}{8024815456} b_{3} \\
& m_{1,4}=\frac{3}{68} b_{1}-\frac{1244724001}{21126554976} b_{3}+\frac{752806667}{21126554976} b_{4} \\
& m_{1,5}=\frac{49579087}{10149031312} b_{3}-\frac{49579087}{10149031312} b_{4} \\
& m_{1,6}=-\frac{1}{784} b_{4}+\frac{1}{784} b_{3} \\
& m_{2,2}=\frac{3481}{3264} b_{1}+\frac{9258282831623875}{7669235228057664} b_{3}+\frac{236024329996203}{1278205871342944} b_{4} \\
& m_{2,3}=-\frac{59}{408} b_{1}-\frac{29294615794607}{29725717938208} b_{3}-\frac{2944673881023}{29725717938208} b_{4} \\
& m_{2,4}=-\frac{59}{1088} b_{1}+\frac{260297319232891}{2556411742685888} b_{3}-\frac{60834186813841}{1278205871342944} b_{4} \\
& m_{2,5}=-\frac{1328188692663}{37594290333616} b_{3}+\frac{1328188692663}{37594290333616} b_{4} \\
& m_{2,6}=-\frac{8673}{2904112} b_{3}+\frac{8673}{2904112} b_{4} \\
& m_{3,3}=\frac{1}{51} b_{1}+\frac{59}{192} b_{2}+\frac{13777050223300597}{26218083221499456} b_{4}+\frac{564461}{13384296} b_{5}+\frac{378288882302546512209}{270764341349677687456} b_{3} \\
& m_{3,4}=\frac{1}{136} b_{1}-\frac{125059}{743572} b_{5}-\frac{4836340090442187227}{5525802884687299744} b_{3}-\frac{17220493277981}{89177153814624} b_{4} \\
& m_{3,5}=-\frac{10532412077335}{42840005263888} b_{4}+\frac{1613976761032884305}{7963657098519931984} b_{3}+\frac{564461}{4461432} b_{5} \\
& m_{3,6}=-\frac{960119}{1280713392} b_{4}-\frac{3391}{6692148} b_{5}+\frac{33235054191}{26452850508784} b_{3} \\
& m_{4,4}=\frac{3}{1088} b_{1}+\frac{507284006600757858213}{475219048083107777984} b_{3}+\frac{1869103}{2230716} b_{5}+\frac{1}{24} b_{6}+\frac{1950062198436997}{3834617614028832} b_{4} \\
& m_{4,5}=-\frac{4959271814984644613}{20965546238960637264} b_{3}-\frac{1}{6} b_{6}-\frac{15998714909649}{37594290333616} b_{4}-\frac{375177}{743572} b_{5} \\
& m_{4,6}=-\frac{368395}{2230716} b_{5}+\frac{752806667}{539854092016} b_{3}+\frac{1063649}{8712336} b_{4}+\frac{1}{8} b_{6} \\
& m_{5,5}=\frac{8386761355510099813}{128413970713633903242} b_{3}+\frac{2224717261773437}{2763180339520776} b_{4}+\frac{5}{6} b_{6}+\frac{1}{24} b_{7}+\frac{280535}{371786} b_{5} \\
& m_{5,6}=-\frac{35039615}{213452232} b_{4}-\frac{1}{6} b_{7}-\frac{13091810925}{13226425254392} b_{3}-\frac{1118749}{2230716} b_{5}-\frac{1}{2} b_{6} \\
& m_{6,6}=\frac{3290636}{80044587} b_{4}+\frac{5580181}{6692148} b_{5}+\frac{5}{6} b_{7}+\frac{1}{24} b_{8}+\frac{660204843}{13226425254392} b_{3}+\frac{3}{4} b_{6}
\end{aligned}
$$

The corresponding right boundary closure is obtained by replacing $b_{i} \rightarrow$ $b_{N+1-i}$ for $i=1, \ldots, 8$ followed by a permutation of both rows and columns. Let $m_{i, j}$ be the entry at row $i$ and column $j$ in $M^{(b)}$. The matrix $M^{(b)}$ is symmetric, which means that it is completely defined by the entries on and above the main diagonal, i.e., $m_{j, i}=m_{i, j}, i=1, \ldots, N, \quad j=i, \ldots, N$. 


\section{References}

[1] Nord2000. comprehensive outdoor sound propagation model. part 2: Propagation in an atmosphere with refraction. Technical Report 1851/00, Nordic Noise Group \& Nordic Road Administration, 2006.

[2] A. Bamberger, B. Engquist, L. Halpern, and P. Joly. Higher order paraxial wave equation approximations in heterogeneous media. SIAM Journal of Applied Mathematics, 48:129-154, 1988.

[3] D. Appelö and T. Colonius. A high-order super-grid-scale absorbing layer and its application to linear hyberbolic systems. J. Comput. Phys., $228: 4200-4217,2009$

[4] M. H. Carpenter, D. Gottlieb, and S. Abarbanel. Time-stable boundary conditions for finite-difference schemes solving hyperbolic systems: Methodology and application to high-order compact schemes. J. Comput. Phys., 111(2), 1994.

[5] M. H. Carpenter, J. Nordström, and D. Gottlieb. A Stable and Conservative Interface Treatment of Arbitrary Spatial Accuracy. J. Comput. Phys., 148, 1999.

[6] Carl de Boor. A Practical Guide to Splines. Springer-Verlag, New York, 1978.

[7] Peter Diener, Ernst Nils Dorband, Erik Schnetter, and Manuel Tiglio. Optimized high-order derivative and dissipation operators satisfying summation by parts, and applications in three-dimensional multi-block evolutions. J. Sci. Comput., 32(1):109-145, 2007.

[8] J. J. Embrechts. Broad spectrum diffusion model for room acoustics raytracing algorithms. The Journal of the Acoustical Society of America, 107(4):2068-2081, 2000.

[9] B. Engquist and A. Majda. Absorbing boundary conditions for the numerical simulation of waves. Mathematics of Computation, 31:629$651,1977$.

[10] B. Gustafsson. The convergence rate for difference approximations to general mixed initial boundary value problems. SIAM J. Numer. Anal., 18(2):179-190, Apr. 1981.

[11] B. Gustafsson, H.-O. Kreiss, and J. Oliger. Time dependent problems and difference methods. John Wiley \& Sons, Inc., 1995.

[12] E. Hairer, S.P. Nørsett, and G.Wanner. Solving Ordinary Differential Equations I. Springer Verlag, 1993. 
[13] J. Hicken and D. Zingg. Superconvergent functional estimates from summation-by-parts finite-difference discretizations. SIAM Journal on Scientific Computing, 33(2):893-922, 2011.

[14] J. E. Hicken and D. W. Zingg. Parallel newton-krylov solver for the euler equations discretized using simultaneous approximation terms. AIAA Journal, 46(11):2773-2786, 2013/02/09 2008.

[15] Jason E. Hicken and David W. Zingg. Aerodynamic optimization algorithm with integrated geometry parameterization and mesh movement. AIAA Journal, 48(2):400-413, 2013/02/09 2010.

[16] J.E. Hicken. Output error estimation for summation-by-parts finitedifference schemes. Journal of Computational Physics, 231(9):3828 $3848,2012$.

[17] Maarten Hornikx and Jens Forsse'n. Modelling of sound propagation to three-dimensional urban courtyards using the extended fourier pstd method. Applied Acustics, 72:665-676, 2011.

[18] R. Jeltsch. Multistep Methods Using Higher Derivatives and Damping at Infinity. Math. Comp., 31:124-138, 1977.

[19] F.B. Jensen, W.A. Kuperman, M.B. Porter, and H. Schmidt. Computational Ocean Acoustics. AIP Press, New York, 1994.

[20] I. Karasalo and A. Sundström. JEPE - a high-order PE-model for rangedependent fluid media. In Proc. 3rd European Conference on Underwater Acoustics, pages 189-194, Heraklion, Crete, Greece, 1996.

[21] H.-O. Kreiss and G. Scherer. Finite element and finite difference methods for hyperbolic partial differential equations. Mathematical Aspects of Finite Elements in Partial Differential Equations., Academic Press, Inc., 1974.

[22] Heinz-Otto Kreiss and Joseph Oliger. Comparison of accurate methods for the integration of hyperbolic equations. Tellus XXIV, 3, 1972.

[23] Samuli Laine, Samuel Siltanen, Tapio Lokki, and Lauri Savioja. Accelerated beam tracing algorithm. Applied Acoustics, 70(1):172 - 181, 2009 .

[24] Conny Larsson. Weather effects on outdoor sound propagation. International Journal of Acoustics and Vibration, 5, 2000.

[25] E. Larsson and L. Abrahamsson. Helmholtz and parabolic equation solutions to a benchmark problem in ocean acoustics. J. Acoust. Soc. Am., 113:2446-2454, 2003. 
[26] L. Lehner, O. Reula, and M. Tiglio. Multi-block simulations in general relativity: high-order discretizations, numerical stability and applications. Classical Quantum Gravity, 22:5283-5321, 2005.

[27] K. Mattsson. Boundary procedures for summation-by-parts operators. Journal of Scientific Computing, 18:133-153, 2003.

[28] K. Mattsson. Summation by parts operators for finite difference approximations of second-derivatives with variable coefficients. Journal of Scientific Computing, 51:650-682, 2012.

[29] K. Mattsson and M. Almquist. A solution to the stability issues with block norm summation by parts operators. J. Comput. Phys., 253:418$442,2013$.

[30] K. Mattsson, F. Ham, and G. Iaccarino. Stable and accurate wave propagation in discontinuous media. J. Comput. Phys., 227:8753-8767, 2008.

[31] K. Mattsson, F. Ham, and G. Iaccarino. Stable boundary treatment for the wave equation on second-order form. Journal of Scientific Computing, 41:366-383, 2009.

[32] K. Mattsson and J. Nordström. Summation by parts operators for finite difference approximations of second derivatives. J. Comput. Phys., 199(2):503-540, 2004.

[33] K. Mattsson and F. Parisi. Stable and accurate second-order formulation of the shifted wave equation. Commun. Comput. Phys., 7:103-137, 2010 .

[34] K. Mattsson, M. Svärd, M.H. Carpenter, and J. Nordström. High-order accurate computations for unsteady aerodynamics. Computers $\mathscr{E}$ Fluids, 36:636-649, 2006.

[35] K. Mattsson, M. Svärd, and M. Shoeybi. Stable and accurate schemes for the compressible navier-stokes equations. J. Comput. Phys., 227(4):2293-2316, 2008.

[36] J. Nordström and M. H. Carpenter. Boundary and interface conditions for high-order finite-difference methods applied to the Euler and NavierStokes equations. J. Comput. Phys., 148:341-365, 1999.

[37] J. Nordström and M. H. Carpenter. High-order finite difference methods, multidimensional linear problems, and curvilinear coordinates. $J$. Comput. Phys., 173:149-174, 2001.

[38] J. Nordström and J. Gong. A stable hybrid method for hyperbolic problems. J. Comput. Phys., 212:436-453, 2006. 
[39] J. Nordström, K. Mattsson, and R.C. Swanson. Boundary conditions for a divergence free velocity-pressure formulation of the incompressible navier-stokes equations. J. Comput. Phys., 225:874-890, 2007.

[40] S. Parakkal, KE. Gilbert, D. Xiao, and HE. Bass. A generalized polar coordinate method for sound propagation over large-scale irregular terrain. J Acoust Soc Am., 128(5):2573-2580, 2010.

[41] T. Van Renterghem and D. Botteldooren. Prediction-step staggered-intime fdtd: An efficient numerical scheme to solve the linearised equations of fluid dynamics in outdoor sound propagation. Applied Acustics, 68:201-216, 2007.

[42] A. Sundström. Energy-conserving parabolic wave equations. FOA Report C20895-2.7, National Defence Research Establishment, Stockholm, 1992.

[43] M. Svärd. On coordinate transformation for summation-by-parts operators. Journal of Scientific Computing, 20(1), 2004.

[44] M. Svärd, M. H. Carpenter, and J. Nordström. A stable high-order finite difference scheme for the compressible Navier-Stokes equations, no-slip wall boundary conditions. J. Comput. Physics, 227:4805-4824, May 2008.

[45] M. Svärd and J. Nordström. On the order of accuracy for difference approximations of initial-boundary value problems. J. Comput. Physics, 218:333-352, October 2006.

[46] Henry Weinberg and Robert Burridge. Horizontal ray theory for ocean acoustics. The Journal of the Acoustical Society of America, 55(1):63$79,1974$.

[47] Chang-Fa Yang, Boau-Cheng Wu, and Chuen-Jyi Ko. A ray-tracing method for modeling indoor wave propagation and penetration. Antennas and Propagation, IEEE Transactions on, 46(6):907-919, 1998.

[48] K.S. Yee. Numerical solution of initial boundary value problems involving Maxwell's equations in isotropic media. IEEE Trans. Antennas Propag., 14:302-307, 1966. 